University of Nebraska - Lincoln

DigitalCommons@University of Nebraska - Lincoln

Faculty Publications from Nebraska Center for Materials and Nanoscience

Materials and Nanoscience, Nebraska Center for (NCMN)

August 1999

\title{
Elastic wave propagation and scattering in heterogeneous, anisotropic media: Textured polycrystalline materials
}

Joseph A. Turner

University of Nebraska - Lincoln, jaturner@unl.edu

Follow this and additional works at: https://digitalcommons.unl.edu/cmrafacpub

Part of the Nanoscience and Nanotechnology Commons

Turner, Joseph A., "Elastic wave propagation and scattering in heterogeneous, anisotropic media: Textured polycrystalline materials" (1999). Faculty Publications from Nebraska Center for Materials and Nanoscience. 60.

https://digitalcommons.unl.edu/cmrafacpub/60

This Article is brought to you for free and open access by the Materials and Nanoscience, Nebraska Center for (NCMN) at DigitalCommons@University of Nebraska - Lincoln. It has been accepted for inclusion in Faculty Publications from Nebraska Center for Materials and Nanoscience by an authorized administrator of DigitalCommons@University of Nebraska - Lincoln. 


\title{
Elastic wave propagation and scattering in heterogeneous, anisotropic media: Textured polycrystalline materials
}

\author{
Joseph A. Turner \\ Department of Engineering Mechanics, W317.4 Nebraska Hall, University of Nebraska-Lincoln, \\ Lincoln, Nebraska 68588-0526
}

(Received 23 September 1998; accepted for publication 3 May 1999)

\begin{abstract}
The propagation of elastic waves through heterogeneous, anisotropic media is considered. Appropriate ensemble averaging of the elastic wave equation leads to the Dyson equation which governs the mean response of the field. The Dyson equation is given here in terms of anisotropic elastic Green's dyadics for the medium with and without heterogeneities. The solution of the Dyson equation for the mean response is given for heterogeneities that are weak. The formalism is further specified for the case of equiaxed cubic polycrystalline metals with a single aligned axis. The Green's dyadics in this case are those for a transversely isotropic medium. Simple expressions for the attenuations of the shear horizontal, quasicompressional, and quasishear waves are given in terms of integrations on the unit circle. The derived expressions are limited to frequencies below the geometric optics limit, but give the attenuations in a direct manner. Comparisons with previous results are also discussed. It is anticipated that a similar approach is necessary for the study of wave propagation in complex anisotropic materials such as fiber-reinforced composites. In addition, the results are applicable to diffuse ultrasonic inspection of textured polycrystalline media. (C) 1999 Acoustical Society of America. [S0001-4966(99)04008-4]
\end{abstract}

PACS numbers: 43.20.Bi, 43.20.Gp, 43.35.Cg [DEC]

\section{INTRODUCTION}

The study of wave propagation and scattering of elastic waves in heterogeneous, anisotropic media is related to nondestructive testing, materials characterization and acoustic emission of many important materials. Examples include polycrystalline media with texture, fiber-reinforced composites, and extruded metal-matrix composites. Elastic waves which propagate through such media lose energy due to scattering from the heterogeneous structure of the material. This scattering may be characterized by the attenuation of the medium. If the medium is statistically isotropic, the attenuation is independent of propagation direction. In an anisotropic medium the scattering attenuation is a function of propagation direction. The analysis of this scattering attenuation is, therefore, more complicated than that of the isotropic case. The study of statistically isotropic media and the corresponding scattering attenuation has received considerable attention. The problem of wave propagation in textured polycrystalline materials has, however, received lesser attention. Stanke and Kino ${ }^{1}$ briefly discuss the applicability of the Keller approximation ${ }^{2}$ to the case of polycrystalline media with texture, although they provide no specific results. Hirsekorn was one of the first to carefully examine the scattering in textured polycrystals as a function of frequency. ${ }^{3}$ The use of a single-sized, spherical grain resulted in unphysical oscillations at higher frequencies. She then extended her theory, using the same perturbation approach, to determine the directional dependence of the phase velocity and attenuation of the three wave types. ${ }^{4}$ The problem was considered more recently by Ahmed and Thompson. ${ }^{5}$ They also examined the case of polycrystalline grains with an aligned axis and developed algebraic equations governing the wave number. The roots of these equations, found numerically, deter- mined the phase velocity and scattering attenuation. Their results were applicable for all frequencies from the Rayleigh limit to the geometric optics limit due to their use of the Keller approximation alone without additional approximations. They have also examined correlations defined by both equiaxed grains and grains with elongation. ${ }^{5,6}$ They further noted the relations between the diffuse backscatter and the presence of texture. $^{7}$

The general scattering problem as discussed by these authors and others involves a scattering integral with a Green's function as its kernel. If the medium is statistically isotropic, this Green's function clearly takes the form of the Green's function for the isotropic medium. In the case of statistically anisotropic media this choice is less clear. Stanke and Kino argue that the isotropic Green's function may be used in the analysis for polycrystalline materials with texture. ${ }^{1}$ This argument was the basis of the work by Ahmed and Thompson. ${ }^{5}$ They used the isotropic Green's function as given by Lifshitz and Parkhamovski ${ }^{8}$ to describe the scattering in textured media. In addition, they simplified the analysis by using the polarization directions for the isotropic waves. Examination of the Keller approximation indicates that the choice of Green's function is not so clear. In the original discussion, ${ }^{2}$ a small variable $\varepsilon$ was defined as a measure of the departure of the medium from homogeneity. The Green's function within the scattering integral corresponded to that for the medium for which the heterogeneity was zero. For textured polycrystalline materials, the homogeneous medium is anisotropic. Thus, an anisotropic Green's function may be more appropriate for this and other similar problems. Comparisons between these different solution methods (e.g., isotropic Green's function with and without anisotropic polarizations and anisotropic Green's function) in terms of the 
strength of the anisotropy will not be addressed here.

The use of an anisotropic Green's function for modeling the scattering in statistically anisotropic media is the subject of this article. Here, the problem is formulated in terms of the Dyson equation as discussed by Frisch ${ }^{9}$ and Weaver. ${ }^{10}$ The Dyson equation is easily solved in the spatial Fourier transform domain within the limits of the first-order smoothing approximation (FOSA), or Keller ${ }^{2}$ approximation. A further approximation is also made which restricts the results to frequencies below the high-frequency geometric optics limit. The problem is further specified for the case of cubic polycrystalline grains with one aligned axis and with the other two axes randomly oriented. In this case, the anisotropy reduces to that of transverse isotropy. The result is the attenuation as a function of direction and frequency for the shear horizontal, quasicompressional, and quasishear waves. In particular, the angular dependence of the attenuations in the Rayleigh limit is obtained explicitly. Outside the Rayleigh limit, simple expressions for the attenuations of the shear horizontal, quasicompressional, and quasishear waves are derived in terms of integrations on the unit circle. The results are quantitatively similar to those of Ahmed and Thompson, ${ }^{5}$ but are given here in a more direct manner. Differences in the angular dependence are also seen due to the use of the anisotropic Green's function here.

The present formulation also allows the extension to the full multiple scattering problem to be made in a straightforward manner. Such an extension in terms of radiative transfer and diffusion has been discussed previously for the statistically isotropic case using similar methods. ${ }^{10-13}$ Although the present application is for textured metals, the formalism is sufficiently general to be applied to other heterogeneous media with statistical anisotropy such as fiber-reinforced composites and extruded metal-matrix composites.

In the next section, the Dyson equation is discussed in terms of the appropriate Green's dyadics. The formalism is developed for a general anisotropic material without reference to a particular symmetry class. The solution of the mean response is further reduced in the succeeding section for the case of a transversely isotropic medium. The elastic modulus tensor is specified for this case and expressions for the attenuation of each wave type are given. Finally, the covariance tensor of the elastic moduli fluctuations is further specified for that of cubic polycrystals with texture and solutions for the case of stainless steel with aligned [001] axes are given.

\section{PRELIMINARY ELASTODYNAMICS}

The equation of motion for the elastodynamic response of an infinite, linear-elastic material to deformation is given in terms of the Green's dyadic by

$$
\begin{gathered}
\left\{-\delta_{j k} \frac{\partial^{2}}{\partial t^{2}}+\frac{\partial}{\partial x_{i}} C_{i j k l}(\mathbf{x}) \frac{\partial}{\partial x_{l}}\right\} G_{k \alpha}\left(\mathbf{x}, \mathbf{x}^{\prime} ; t\right) \\
=\delta_{j \alpha} \delta^{3}\left(\mathbf{x}-\mathbf{x}^{\prime}\right) \delta(t) .
\end{gathered}
$$

The second-rank Green's dyadic, $G_{k \alpha}\left(\mathbf{x}, \mathbf{x}^{\prime} ; t\right)$, defines the response at location $\mathbf{x}$ in the $k$ th direction to a unit im- pulse at location $\mathbf{x}^{\prime}$ in the $\alpha$ th direction. The moduli are spatially variable and density is assumed uniform throughout. The units in Eq. (1) have been chosen such that the density is unity. The moduli are assumed to be spatially heterogeneous and of the form $C_{i j k l}(\mathbf{x})=C_{i j k l}^{0}+\delta C_{i j k l}(\mathbf{x})$. Thus, the moduli have the form of average moduli, $C_{i j k l}^{0}$ $=\left\langle C_{i j k l}(\mathbf{x})\right\rangle$, plus a fluctuation about this mean, $\delta C_{i j k l}(\mathbf{x})$. The fluctuations are assumed to have zero mean, $\left\langle\delta C_{i j k l}(\mathbf{x})\right\rangle=0$. The mean moduli are not necessarily isotropic - the material may have global anisotropy. The covariance of the moduli is represented by an eighth-rank tensor

$$
\Lambda(\mathbf{x}-\mathbf{y})_{\alpha \beta \gamma \delta}^{i j k l}=\left\langle\delta C_{i j k l}(\mathbf{x}) \delta C_{\alpha \beta \gamma \delta}(\mathbf{y})\right\rangle .
$$

The covariance, $\boldsymbol{\Lambda}$, is given as a function of the difference between two vectors, $\mathbf{x}-\mathbf{y}$. This assumption implies that the medium is statistically homogeneous. However, the additional assumption of statistical isotropy (that $\mathbf{\Lambda}$ is a function of $|\mathbf{x}-\mathbf{y}|$ ) is not made here. The power spectral density of the moduli fluctuations, $\tilde{\Lambda}$, is given by the Fourier transform of the covariance

$$
\tilde{\Lambda}(\mathbf{p})_{\alpha \beta \gamma \delta}^{i j k l}=\int \Lambda(\mathbf{r})_{\alpha \beta \gamma \delta}^{i j k l} e^{-i \mathbf{r} \cdot \mathbf{p}} d^{3} r .
$$

Defining a temporal Fourier transform pair for the functions $f(t)$ and $\widetilde{f}(\omega)$ by

$$
\begin{aligned}
& \widetilde{f}(\omega)=\int f(t) e^{i \omega t} d t, \\
& f(t)=\frac{1}{2 \pi} \int \tilde{f}(\omega) e^{-i \omega t} d \omega,
\end{aligned}
$$

allows Eq. (1) to be transformed as

$$
\begin{gathered}
\left\{\omega^{2} \delta_{j k}+C_{i j k l}^{0} \frac{\partial}{\partial x_{i}} \frac{\partial}{\partial x_{l}}+\frac{\partial}{\partial x_{i}} \delta C_{i j k l}(\mathbf{x}) \frac{\partial}{\partial x_{l}}\right\} G_{k \alpha}\left(\mathbf{x}, \mathbf{x}^{\prime}, \omega\right) \\
=\delta_{j \alpha} \delta^{3}\left(\mathbf{x}-\mathbf{x}^{\prime}\right) .
\end{gathered}
$$

The random nature of the medium suggests that the Green's function, $\mathbf{G}$, is of little value as it will also be a random function. The interesting quantities are instead those related to the statistics of the response. These statistics include the mean response, $\langle\mathbf{G}\rangle$, and the covariance of the response, $\left\langle\mathbf{G G}^{*}\right\rangle$, with the $*$ denoting a complex conjugate. This article is devoted to examination of the mean response with corresponding phase velocities and attenuations.

Wave propagation and scattering problems of this sort do not lend themselves to solution by perturbation methods. As Frisch points out, these solutions do not converge. ${ }^{9}$ Instead Frisch used diagrammatic methods for solution of the mean response. ${ }^{9}$ The mean response, $\langle\mathbf{G}\rangle$, is governed by the Dyson equation which is given by ${ }^{9,10}$

$$
\begin{aligned}
\left\langle G_{i \alpha}\left(\mathbf{x}, \mathbf{x}^{\prime}\right)\right\rangle= & G_{i \alpha}^{0}\left(\mathbf{x}, \mathbf{x}^{\prime}\right)+\iint G_{i \beta}^{0}(\mathbf{x}, \mathbf{y}) M_{\beta j}(\mathbf{y}, \mathbf{z}) \\
& \times\left\langle G_{j \alpha}\left(\mathbf{z}, \mathbf{x}^{\prime}\right)\right\rangle d^{3} y d^{3} z .
\end{aligned}
$$

In Eq. (7), the quantity $\mathbf{G}^{0}$ is the bare Green's dyadic. It defines the response of the medium without 
heterogeneities - the solution of Eq. (6) with $\delta C_{i j k l}(\mathbf{x})=0$. The second-rank tensor $\mathbf{M}$ is the mass or self-energy operator. ${ }^{9}$ The Dyson equation, Eq. (7), is easily solved in Fourier transform space under the assumption of statistical homogeneity. The spatial Fourier transform pair for $\mathbf{G}^{0}$ is given by

$$
\begin{aligned}
G_{i \alpha}^{0}(\mathbf{p}) \delta^{3}(\mathbf{p}-\mathbf{q})= & \frac{1}{(2 \pi)^{3}} \iint G_{i \alpha}^{0}\left(\mathbf{x}, \mathbf{x}^{\prime}\right) \\
& \times e^{-i \mathbf{p} \cdot \mathbf{x}} e^{i \mathbf{q} \cdot \mathbf{x}^{\prime}} d^{3} x d^{3} x^{\prime}, \\
G_{i \alpha}^{0}\left(\mathbf{x}, \mathbf{x}^{\prime}\right)= & \frac{1}{(2 \pi)^{3}} \iint G_{i \alpha}^{0}(\mathbf{p}) \delta^{3}(\mathbf{p}-\mathbf{q}) \\
& \times e^{i \mathbf{p} \cdot \mathbf{x}} e^{-i \mathbf{q} \cdot \mathbf{x}^{\prime}} d^{3} p d^{3} q .
\end{aligned}
$$

The Fourier transforms which define $\langle\mathbf{G}(\mathbf{p})\rangle$ and $\tilde{\mathbf{M}}(\mathbf{p})$ are given by expressions similar to that defining $\mathbf{G}^{0}(\mathbf{p})$. The assumption of statistical homogeneity ensures that they are functions of a single wave vector in Fourier space. The Dyson equation can then be spatially Fourier transformed and solved for $\langle\mathbf{G}(\mathbf{p})\rangle$. The result is

$$
\langle\mathbf{G}(\mathbf{p})\rangle=\left[\mathbf{G}^{0}(\mathbf{p})^{-1}-\tilde{\mathbf{M}}(\mathbf{p})\right]^{-1},
$$

where $\tilde{\mathbf{M}}$ is the spatial transform of the self-energy. The Dyson equation is exact and describes the mean response of the medium. The main difficulty in the solution of Eq. (10) is the representation of $\mathbf{M}$. Approximations of $\mathbf{M}$ are often necessary to obtain closed-form solutions of Eq. (10). The selfenergy, M, can be written as an expansion in powers of moduli fluctuations. Approximation of $\mathbf{M}$ can then be made to first order using the first term in such an expansion. Frisch discusses the equivalence of this technique, which he called the first-order smoothing approximation (FOSA), ${ }^{9}$ and the Keller approximation. ${ }^{2}$ The FOSA expression for $\mathbf{M}$ is given by ${ }^{10}$

$$
\begin{aligned}
M_{\beta j}(\mathbf{y}, \mathbf{z}) \approx & \left\langle\frac{\partial}{\partial y_{\alpha}} \delta C_{\alpha \beta \gamma \delta}(\mathbf{y})\right. \\
& \left.\times \frac{\partial}{\partial y_{\delta}} G_{\gamma k}^{0}(\mathbf{y}, \mathbf{z}) \frac{\partial}{\partial z_{i}} \delta C_{i j k l}(\mathbf{z}) \frac{\partial}{\partial z_{l}}\right\rangle .
\end{aligned}
$$

Such an approximation is assumed valid as long as the fluctuations, $\delta \mathbf{C}$, are not too large. The spatial Fourier transform, as defined by Eq. (8), of the self-energy, M, is then formulated. Manipulation of this integration allows it to be reduced to ${ }^{10}$

$$
\tilde{M}_{\beta j}(\mathbf{p})=\int d^{3} s G_{\gamma k}^{0}(\mathbf{s}) p_{\alpha} p_{l} s_{\delta} s_{i} \tilde{\Lambda}(\mathbf{p}-\mathbf{s})_{\alpha \beta \gamma \delta}^{i j k l} .
$$

Thus, the transform of the self-energy can be written as a convolution between the bare Green's dyadic and the Fourier transform of the covariance of the moduli fluctuations. This expression, Eq. (12), and the Dyson equation, Eq. (10), are the primary results of this section which are used in the remainder of the article. The components of $\tilde{\mathbf{M}}$, as discussed in Sec. III, are used to calculate the phase velocity and attenuation of the various propagation modes. Equation (12) is identical to that given by Weaver for a statistically isotropic medium. However, the use of an anisotropic Green's dyadic for representing $\mathbf{G}^{0}$, which is discussed below, is the main new result here. The abbreviated presentation given in this section serves as a reminder of the general procedure for the description of the mean response. The Dyson equation, Eq. (10), and the expression for the self-energy, Eq. (12), will be used below for the derivation of the attenuations. Readers interested in further details of the scattering theory are referred to the articles of Karal and Keller, ${ }^{2}$ Frisch, ${ }^{9}$ Stanke and Kino, ${ }^{1}$ and Weaver. ${ }^{10}$

\section{GREEN'S DYADIC FOR TRANSVERSELY ISOTROPIC MEDIA}

The solution of the Dyson equation, Eq. (10), for the mean response requires the Green's dyadic for the bare medium. The bare Green's dyadic, $\mathbf{G}^{0}$, is defined as the solution of the equation of motion, Eq. (6), without heterogeneities $\left[\delta C_{i j k l}(\mathbf{x})=0\right]$. The emphasis here is on anisotropic media with heterogeneities. Thus, the $\mathbf{G}^{0}$ required is that for an anisotropic medium. The lowest possible anisotropic symmetry class to be considered is that of a medium with a single symmetry axis. Although this is the simplest case of global anisotropy, relevant transversely isotropic or uniaxial materials include fiber-reinforced composites and polycrystalline media with fiber textures. These types of media have a single symmetry axis defined here by the unit vector, $\hat{\mathbf{n}}$. This direction is termed the "fiber", direction although the medium may not be composed of any fibers. The fourth-rank elastic moduli tensor, $\mathbf{C}$, in a transversely isotropic medium is a function of $\hat{\mathbf{n}}$ and is written

$$
\begin{aligned}
C_{i j k l}= & \lambda_{\perp} \delta_{i j} \delta_{k l}+\mu_{\perp}\left(\delta_{i k} \delta_{j l}+\delta_{i l} \delta_{j k}\right)+A\left(\delta_{i j} \hat{n}_{k} \hat{n}_{l}\right. \\
& \left.+\delta_{k l} \hat{n}_{i} \hat{n}_{j}\right)+B\left(\delta_{i k} \hat{n}_{j} \hat{n}_{l}+\delta_{i l} \hat{n}_{j} \hat{n}_{k}+\delta_{j k} \hat{n}_{i} \hat{n}_{l}\right. \\
& \left.+\delta_{j l} \hat{n}_{i} \hat{n}_{k}\right)+D \hat{n}_{i} \hat{n}_{j} \hat{n}_{k} \hat{n}_{l} .
\end{aligned}
$$

The above elastic constants are defined as $A=\nu-\lambda_{\perp}, B$ $=\mu_{\|}-\mu_{\perp}$, and $D=\lambda_{\perp}+2 \mu_{\perp}+\lambda_{\|}+2 \mu_{\|}-2\left(\nu+2 \mu_{\|}\right)$. The elastic moduli, $\lambda_{\|}+2 \mu_{\|}, \lambda_{\perp}+2 \mu_{\perp}, \mu_{\perp}, \mu_{\|}$, and $\nu$ can be defined alternatively as $C_{11}, C_{33}, C_{44}, C_{66}$, and $C_{13}$, respectively. $^{14}$

Substitution of this form for $\mathbf{C}$ into the equation of motion, Eq. (6), gives in direct notation

$$
\begin{aligned}
\left\{\omega^{2} \mathbf{I}\right. & -p^{2}\left[\left(\mu_{\perp}+B(\hat{\mathbf{p}} \cdot \hat{\mathbf{n}})^{2}\right) \mathbf{I}+\hat{\mathbf{p}} \hat{\mathbf{p}}\left(\lambda_{\perp}+\mu_{\perp}\right)\right. \\
& +\hat{\mathbf{n}} \hat{\mathbf{n}}\left(B+D(\hat{\mathbf{p}} \cdot \hat{\mathbf{n}})^{2}\right)+(A+B)(\hat{\mathbf{p}} \cdot \hat{\mathbf{n}}) \\
& \times(\hat{\mathbf{p}} \hat{\mathbf{n}}+\hat{\mathbf{n}} \hat{\mathbf{p}})]\} \cdot \mathbf{G}^{0}(\mathbf{p}, \hat{\mathbf{n}})=\mathbf{I},
\end{aligned}
$$

where $\mathbf{p}$ is the wave vector with magnitude, $p$, and direction $\hat{\mathbf{p}}$.

The above equation can be written in terms of the wave matrix, $\mathbf{N}$, by $^{14}$

$$
N_{j k} G_{k \alpha}(\mathbf{p}, \omega)=\delta_{j \alpha} \text {. }
$$

The eigenvectors of $\mathbf{N}$ define the polarization directions for the propagating waves. ${ }^{14}$ These eigenvectors can be found directly from the wave matrix, N. Explicit expressions 
of the directions of these eigenvectors are required for the results which follow. For this reason, an alternative method of determining the eigenvectors is given here.

The shear horizontal wave in a transversely isotropic medium is known to be polarized perpendicular to the plane defined by $\hat{\mathbf{p}}$ and $\hat{\mathbf{n}}$. This vector, $\hat{\mathbf{u}}_{1}$, is given by

$$
\hat{\mathbf{u}}_{1}=\frac{\hat{\mathbf{p}} \times \hat{\mathbf{n}}}{|\hat{\mathbf{p}} \times \hat{\mathbf{n}}|}=\frac{\hat{\mathbf{p}} \times \hat{\mathbf{n}}}{\sin \Theta},
$$

where $\Theta$ defines the angle between the $\hat{\mathbf{p}}$ and $\hat{\mathbf{n}}$. The identity dyadic can then be expanded as $\mathbf{I}=\hat{\mathbf{u}}_{1} \hat{\mathbf{u}}_{1}+\left(\mathbf{I}-\hat{\mathbf{u}}_{1} \hat{\mathbf{u}}_{1}\right)$. Use of the identity ${ }^{15}$

$$
\begin{aligned}
(\hat{\mathbf{p}} \cdot \hat{\mathbf{n}}) & (\hat{\mathbf{p}} \hat{\mathbf{n}}+\hat{\mathbf{n}} \hat{\mathbf{p}}) \\
= & \hat{\mathbf{u}}_{1} \hat{\mathbf{u}}_{1}\left(1-(\hat{\mathbf{p}} \cdot \hat{\mathbf{n}})^{2}\right)-\mathbf{I}\left(1-(\hat{\mathbf{p}} \cdot \hat{\mathbf{n}})^{2}\right)+\hat{\mathbf{n}} \hat{\mathbf{n}}+\hat{\mathbf{p}} \hat{\mathbf{p}},
\end{aligned}
$$

allows the last term in Eq. (14) to be expanded. The result is

$$
\begin{aligned}
\left\{\hat{\mathbf{u}}_{1} \hat{\mathbf{u}}_{1}\right. & {\left[g_{\mathrm{SH}}^{0}(\mathbf{p})\right]^{-1}+\left(\mathbf{I}-\hat{\mathbf{u}}_{1} \hat{\mathbf{u}}_{1}\right) \omega^{2} } \\
& \left.-p^{2}\left[\left(\mathbf{I}-\hat{\mathbf{u}}_{1} \hat{\mathbf{u}}_{1}\right) Q+P \hat{\mathbf{p}} \hat{\mathbf{p}}+E \hat{\mathbf{n}} \hat{\mathbf{n}}\right]\right\} \cdot \mathbf{G}^{0}(\mathbf{p}, \hat{\mathbf{n}})=\mathbf{I},
\end{aligned}
$$

where the shear horizontal dispersion relation is

$$
\left[g_{\mathrm{SH}}^{0}(\mathbf{p})\right]^{-1}=\omega^{2}-p^{2}\left(\mu_{\perp}+B(\hat{\mathbf{p}} \cdot \hat{\mathbf{n}})^{2}\right) .
$$

The quantities $Q, P$, and $E$ in Eq. (18) are defined by

$$
\begin{aligned}
& Q=\mu_{\perp}+B(\hat{\mathbf{p}} \cdot \hat{\mathbf{n}})^{2}-(A+B)\left(1-(\hat{\mathbf{p}} \cdot \hat{\mathbf{n}})^{2}\right), \\
& P=\lambda_{\perp}+\mu_{\perp}+A+B=\nu+\mu_{\|}, \\
& E=A+2 B+D(\hat{\mathbf{p}} \cdot \hat{\mathbf{n}})^{2} .
\end{aligned}
$$

The quasi $\mathrm{P}$ and $\mathrm{SV}$ waves are polarized in directions defined by $\hat{\mathbf{u}}_{2}$ and $\hat{\mathbf{u}}_{3}$ both of which lie in the $\hat{\mathbf{p}}-\hat{\mathbf{n}}$ plane. They form an orthonormal basis with $\hat{\mathbf{u}}_{1}$ such that $\hat{\mathbf{u}}_{3}=\hat{\mathbf{u}}_{1} \times \hat{\mathbf{u}}_{2}$. The vector $\hat{\mathbf{u}}_{2}$ is directed at an angle $\psi$ from the propagation direction $\hat{\mathbf{p}}$. Use of the directions $\hat{\mathbf{u}}_{2}$ and $\hat{\mathbf{u}}_{3}$ allows $\mathbf{G}^{0}$ to be diagonalized. Thus $\mathbf{I}-\hat{\mathbf{u}}_{1} \hat{\mathbf{u}}_{1}=\hat{\mathbf{u}}_{2} \hat{\mathbf{u}}_{2}+\hat{\mathbf{u}}_{3} \hat{\mathbf{u}}_{3}$. The vectors $\hat{\mathbf{p}}$ and $\hat{\mathbf{n}}$ are then written in terms of $\hat{\mathbf{u}}_{2}$ and $\hat{\mathbf{u}}_{3}$ as

$$
\begin{aligned}
& \hat{\mathbf{p}}=\hat{\mathbf{u}}_{2} \cos \psi+\hat{\mathbf{u}}_{3} \sin \psi, \\
& \hat{\mathbf{n}}=\hat{\mathbf{u}}_{2} \cos (\psi+\Theta)+\hat{\mathbf{u}}_{3} \sin (\psi+\Theta) .
\end{aligned}
$$

Substitution into Eq. (18) gives

$$
\begin{aligned}
\left\{\hat{\mathbf{u}}_{1}\right. & \hat{\mathbf{u}}_{1} \\
& {\left[g_{\mathrm{SH}}^{0}(\mathbf{p})\right]^{-1}+\hat{\mathbf{u}}_{2} \hat{\mathbf{u}}_{2}\left[\omega^{2}-p^{2}\left(Q+P \cos ^{2} \psi\right.\right.} \\
& \left.\left.+E \cos ^{2}(\Theta+\psi)\right)\right]+\hat{\mathbf{u}}_{3} \hat{\mathbf{u}}_{3}\left[\omega^{2}-p^{2}\left(Q+P \sin ^{2} \psi+E \sin ^{2}\right.\right. \\
& \times(\Theta+\psi))]-\left(\hat{\mathbf{u}}_{2} \hat{\mathbf{u}}_{3}+\hat{\mathbf{u}}_{3} \hat{\mathbf{u}}_{2}\right)[E \sin (\Theta+\psi) \cos (\Theta+\psi) \\
& +P \sin \psi \cos \psi]\} \cdot \mathbf{G}^{0}(\mathbf{p})=\mathbf{I} .
\end{aligned}
$$

The vectors $\hat{\mathbf{u}}_{2}$ and $\hat{\mathbf{u}}_{3}$, which define the polarizations of the quasi-P and quasi-SV waves, respectively, are eigenvectors of $\mathbf{N}$ and diagonalize $\mathbf{G}^{0}$. Thus, the term in Eq. (23) containing $\hat{\mathbf{u}}_{2} \hat{\mathbf{u}}_{3}+\hat{\mathbf{u}}_{3} \hat{\mathbf{u}}_{2}$ must be zero. This fact establishes a criterion for the angle $\psi$ given by

$$
\tan 2 \psi=\frac{-E \sin 2 \Theta}{P+E \cos 2 \Theta} .
$$

The directions of $\hat{\mathbf{u}}_{2}$ and $\hat{\mathbf{u}}_{3}$ are defined in terms of $\psi$ for later convenience. The above equation for $\psi$, Eq. (24), must be used with care when numerical methods are employed. The value of $\psi$ which satisfies Eq. (24) may correspond to either the quasi-P or quasi-SV wave. It should also be kept in mind that the vectors $\hat{\mathbf{u}}_{2}$ and $\hat{\mathbf{u}}_{3}$ are functions of the direction of propagation, $\hat{\mathbf{p}}$, relative to the fiber direction, $\hat{\mathbf{n}}$. This dependence, $\psi=\psi(\Theta)$, which is evident in Eq. (24), will remain implicit throughout.

The bare Green's dyadic may now be written

$$
\mathbf{G}^{0}(\mathbf{p})=g_{\mathrm{SH}}^{0}(\mathbf{p}) \hat{\mathbf{u}}_{1} \hat{\mathbf{u}}_{1}+g_{\mathrm{qP}}^{0}(\mathbf{p}) \hat{\mathbf{u}}_{2} \hat{\mathbf{u}}_{2}+g_{\mathrm{qSV}}^{0}(\mathbf{p}) \hat{\mathbf{u}}_{3} \hat{\mathbf{u}}_{3} .
$$

The dispersion relations for the bare response of the $\mathrm{SH}$, $\mathrm{qP}$, and $\mathrm{qSV}$ waves are given by

$$
\begin{aligned}
g_{\mathrm{SH}}^{0}(\mathbf{p}) & =\left[\omega^{2}-p^{2}\left(\mu_{\perp}+B \cos ^{2} \Theta\right)\right]^{-1}=\left[\omega^{2}-p^{2} c_{\mathrm{SH}}^{2}\right]^{-1}, \\
g_{\mathrm{qP}}^{0}(\mathbf{p}) & =\left[\omega^{2}-p^{2}\left(Q+P \cos ^{2} \psi+E \cos ^{2}(\Theta+\psi)\right)\right]^{-1} \\
& =\left[\omega^{2}-p^{2} c_{\mathrm{qP}}^{2}\right]^{-1}, \\
g_{\mathrm{qSV}}^{0}(\mathbf{p}) & =\left[\omega^{2}-p^{2}\left(Q+P \sin ^{2} \psi+E \sin ^{2}(\Theta+\psi)\right)\right]^{-1} \\
= & {\left[\omega^{2}-p^{2} c_{\mathrm{qSV}}^{2}\right]^{-1}, }
\end{aligned}
$$

where the angle $\psi$ is defined by Eq. (24) and $Q, P$, and $E$ are defined in Eq. (20). The above expressions also describe the phase velocity, $c_{\beta}$, as a function of propagation direction for each wave type, $\beta$. The imaginary parts of these expressions will be used below and are given by

$$
\operatorname{Im} g_{\beta}^{0}(\mathbf{p})=-\pi \operatorname{sgn}(\omega) \delta\left(\omega^{2}-p^{2} c_{\beta}^{2}\right) .
$$

The mean response of the heterogeneous medium is now given by solution of the Dyson equation.

\section{MEAN RESPONSE}

The mean response, $\langle\mathbf{G}(\mathbf{p})\rangle$, is given by solution of the Dyson equation, Eq. (10), above. The solution of $\langle\mathbf{G}(\mathbf{p})\rangle$ is expressed in terms of $\mathbf{G}^{0}(\mathbf{p})$ and $\tilde{\mathbf{M}}(\mathbf{p})$. Like $\mathbf{G}^{0}$, the mean response and self-energy may be expanded in terms of the orthonormal basis defined by $\hat{\mathbf{u}}_{1}, \hat{\mathbf{u}}_{2}$, and $\hat{\mathbf{u}}_{3}$. Thus,

$$
\begin{gathered}
\langle\mathbf{G}(\mathbf{p})\rangle=g_{\mathrm{SH}}(\mathbf{p}) \hat{\mathbf{u}}_{1} \hat{\mathbf{u}}_{1}+g_{\mathrm{qP}}(\mathbf{p}) \hat{\mathbf{u}}_{2} \hat{\mathbf{u}}_{2}+g_{\mathrm{qSV}}(\mathbf{p}) \hat{\mathbf{u}}_{3} \hat{\mathbf{u}}_{3}, \\
\tilde{\mathbf{M}}(\mathbf{p})=m_{\mathrm{SH}}(\mathbf{p}) \hat{\mathbf{u}}_{1} \hat{\mathbf{u}}_{1}+m_{\mathrm{qP}}(\mathbf{p}) \hat{\mathbf{u}}_{2} \hat{\mathbf{u}}_{2}+m_{\mathrm{qSV}}(\mathbf{p}) \hat{\mathbf{u}}_{3} \hat{\mathbf{u}}_{3},
\end{gathered}
$$

where it is again emphasized that the directions $\hat{\mathbf{u}}_{1}, \hat{\mathbf{u}}_{2}$, and $\hat{\mathbf{u}}_{3}$ are dependent upon the propagation direction $\hat{\mathbf{p}}$.

The dispersion relations for the mean response are then given by the solution of the Dyson equation, Eq. (10), as

$$
\begin{aligned}
g_{\beta}(\mathbf{p}) & =\left[g_{\beta}^{0}(\mathbf{p})^{-1}-m_{\beta}(\mathbf{p})\right]^{-1} \\
& =\left[\omega^{2}-p^{2} c_{\beta}^{2}-m_{\beta}(\mathbf{p})\right]^{-1},
\end{aligned}
$$


for each wave type, $\beta$. This expression for the dispersion relation of the mean response defines the phase velocity and attenuation of each wave type. Solution of

$$
\omega^{2}-p^{2} c_{\beta}^{2}-m_{\beta}(\mathbf{p})=0,
$$

for the wave vector $p$, is required given $\widetilde{\mathbf{M}}$ defined in Eq. (12). The inverse Fourier transform of $\langle\mathbf{G}(\mathbf{p})\rangle$ will be dominated by the poles of the dispersion relations. The phase velocity is given by the real part of $p$ and the attenuation by the imaginary part. Such solutions of Eq. (30) are usually done numerically using root finding techniques. ${ }^{5}$ However, explicit expressions for the attenuation can be determined using an approximation valid below the high-frequency geometric optics limit. In this case, the wave vector $\mathbf{p}$ within the self-energy is approximated as being equal to the bare wave vector. Such an approximation, $m_{\beta}(\mathbf{p}) \approx m_{\beta}\left[\left(\omega / c_{\beta}\right) \hat{\mathbf{p}}\right]$, is sometimes called a Born approximation. ${ }^{1,10}$ This approximation allows the imaginary part of $\mathbf{p}$ to be calculated directly from Eq. (30). The attenuation of each wave type is given by

$$
\alpha_{\beta}(\hat{\mathbf{p}})=-\frac{1}{2 \omega c_{\beta}(\hat{\mathbf{p}})} \operatorname{Im} m_{\beta}\left(\frac{\omega}{c_{\beta}} \hat{\mathbf{p}}\right) .
$$

The final step in this derivation now lies in the expression for the imaginary part of the self-energy. The definition of the self-energy was given by Eq. (12). The form of the self-energy given by Eq. (28) is substituted into Eq. (12). Appropriate inner products allow each component of the self-energy to be determined independently. The wave numbers which appear in Eq. (12) are approximated to the same degree of the Born approximation discussed above. The integration over the magnitude of the wave vector is easily done due to the delta-function form of $\mathbf{G}^{0}(\mathbf{s})$. The delta functions are the result of consideration of the imaginary parts of the dispersion relations given by Eq. (27). The attenuations for the three wave types, each defined by Eq. (31), are finally given by

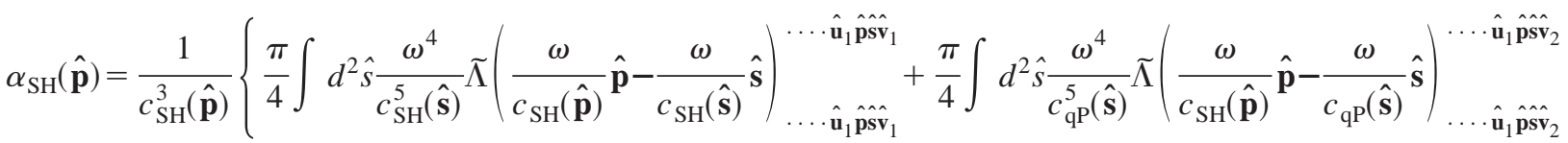

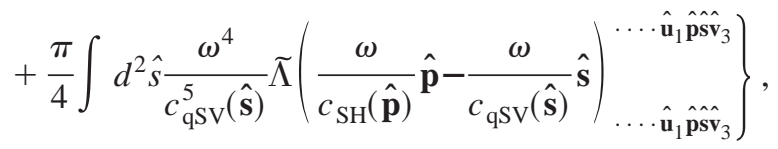

$$
\begin{aligned}
& \alpha_{\mathrm{qP}}(\hat{\mathbf{p}})=\frac{1}{c_{\mathrm{qP}}^{3}(\hat{\mathbf{p}})}\left\{\frac{\pi}{4} \int d^{2} \hat{s} \frac{\omega^{4}}{c_{\mathrm{SH}}^{5}(\hat{\mathbf{s}})} \tilde{\Lambda}\left(\frac{\omega}{c_{\mathrm{qP}}(\hat{\mathbf{p}})} \hat{\mathbf{p}}-\frac{\omega}{c_{\mathrm{SH}}(\hat{\mathbf{s}})} \hat{\mathbf{s}}\right)_{\cdots \hat{\mathbf{u}}_{2} \hat{\mathbf{p}} \hat{\mathbf{v}}_{1}}^{\cdots \hat{\mathbf{u}}_{2} \hat{\mathbf{p}} \hat{\mathbf{v}}_{1}}+\frac{\pi}{4} \int d^{2} \hat{s} \frac{\omega^{4}}{c_{\mathrm{qP}}^{5}(\hat{\mathbf{s}})} \tilde{\Lambda}\left(\frac{\omega}{c_{\mathrm{qP}}(\hat{\mathbf{p}})} \hat{\mathbf{p}}-\frac{\omega}{c_{\mathrm{qP}}(\hat{\mathbf{s}})} \hat{\mathbf{s}}\right)_{\cdots \cdots \hat{\mathbf{u}}_{2} \hat{\mathbf{p}} \hat{\mathbf{s}} \hat{\mathbf{v}}_{2}}^{\cdots \hat{\mathbf{p}} \hat{\mathbf{s}}_{2}}\right.
\end{aligned}
$$

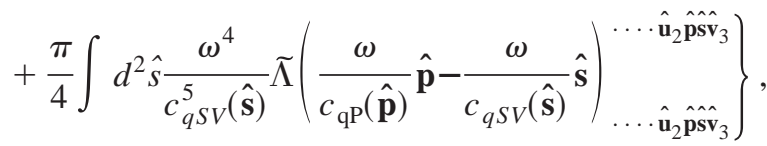

$$
\begin{aligned}
& \alpha_{\mathrm{qSV}}(\hat{\mathbf{p}})=\frac{1}{c_{\mathrm{qSV}}^{3}(\hat{\mathbf{p}})}\left\{\frac{\pi}{4} \int d^{2} \hat{s} \frac{\omega^{4}}{c_{\mathrm{SH}}^{5}(\hat{\mathbf{s}})} \tilde{\Lambda}\left(\frac{\omega}{c_{\mathrm{qSV}}(\hat{\mathbf{p}})} \hat{\mathbf{p}}-\frac{\omega}{c_{\mathrm{SH}}(\hat{\mathbf{s}})} \hat{\mathbf{s}}\right)_{\cdots \hat{\mathbf{u}}_{3} \hat{\mathbf{p}} \hat{\mathbf{v}}_{1}}^{\cdots \hat{\mathbf{u}}_{3} \hat{\mathbf{p}} \hat{\mathbf{v}}_{1}}+\frac{\pi}{4} \int d^{2} \hat{s} \frac{\omega^{4}}{c_{\mathrm{qP}}^{5}(\hat{\mathbf{s}})} \tilde{\Lambda}\left(\frac{\omega}{c_{\mathrm{qSV}}(\hat{\mathbf{p}})} \hat{\mathbf{p}}-\frac{\omega}{c_{\mathrm{qP}}(\hat{\mathbf{s}})} \hat{\mathbf{s}}\right)_{\cdots \cdots \hat{\mathbf{u}}_{3} \hat{\mathbf{p}} \hat{\mathbf{s}} \hat{\mathbf{v}}_{2}}^{\cdots \hat{\mathbf{p s}} \hat{v}_{2}}\right.
\end{aligned}
$$

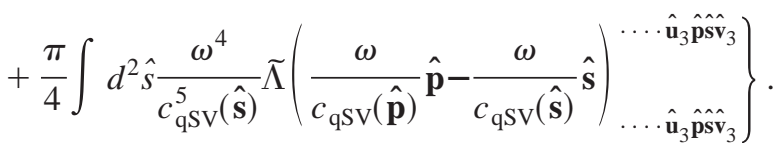

The integrals in the above equations are over the unit sphere defined by $\hat{\mathbf{s}}$. The direction $\hat{\mathbf{p}}$ defines the propagation direction, $\hat{\mathbf{s}}$ is the scattered direction, and the polarization directions, $\hat{\mathbf{u}}$ and $\hat{\mathbf{v}}$. In the above expressions the dependence of the vectors $\hat{\mathbf{u}}$ on $\hat{\mathbf{p}}$ and of $\hat{\mathbf{v}}$ on $\hat{\mathbf{s}}$ is implicit. The argument of the autocorrelation is the difference between the incoming and outgoing propagation directions. The inner products on the autocorrelation of the moduli fluctuations are given in terms of four unit vectors. This inner product is given explic- itly by $\tilde{\Lambda}(\mathbf{q})_{\ldots}^{\cdots} \ldots \hat{\text { unpspsv }}=\tilde{\Lambda}(\mathbf{q})_{\alpha \beta \gamma \delta}^{i j k l} \hat{u}_{\beta} \hat{u}_{k} \hat{p}_{\alpha} \hat{p}_{l} \hat{s}_{i} \hat{s}_{\delta} \hat{v}_{\gamma} \hat{v}_{j}$.

The above expressions for the attenuation are more complicated than those for a statistically isotropic medium considered by Weaver. ${ }^{10}$ They reduce to the forms given there in the case of statistical isotropy. The dependence of wave speed and polarization direction on propagation direction greatly complicates the integrations. In the next section, the covariance is specified for equiaxed cubic polycrystals with texture. 


\section{EQUIAXED POLYCRYSTALLINE MEDIA WITH TEXTURE}

The above formalism is now specified for the particular problem of equiaxed cubic polycrystalline media with one aligned axis. This particular grain structure arises during welding or solidification. In this case the transverse isotropy is the result of alignment of one grain axis in all grains. The other axes are randomly oriented about that axis. Two assumptions are made about the fluctuations of the elastic moduli. The tensorial and spatial components of covariance are first assumed independent. This assumption implies

$$
\widetilde{\Lambda}(\mathbf{p})_{\alpha \beta \gamma \delta}^{i j k l}=\Xi_{\alpha \beta \gamma \delta}^{i j k l} \widetilde{W}(\mathbf{p}),
$$

where $\widetilde{W}(\mathbf{p})$ is the Fourier transform of the spatial correlation function. The grains are also assumed to be equiaxed such that $W(\mathbf{r})=e^{-r / L}$. The correlation length, $L$, is of the order of the grain radius. The Fourier transform is then given by

$$
\widetilde{W}(\mathbf{q})=\frac{L^{3}}{\pi^{2}\left(1+L^{2} q^{2}\right)^{2}} .
$$

The forms of the attenuation given above contain the difference of two vectors, $\widetilde{W}(\mathbf{q})=\widetilde{W}\left(\left[\omega / c_{1}(\Theta)\right] \hat{\mathbf{p}}\right.$ $\left.-\left[\omega / c_{2}\left(\Theta^{\prime}\right)\right] \hat{\mathbf{s}}\right)$ as the argument for $\tilde{\Lambda}(\mathbf{p})$. The form of correlation function is dependent upon magnitude of this vector. Trigonometry reduces the magnitude of this difference to

$$
\begin{aligned}
q^{2} & =\left|\frac{\omega}{c_{1}(\Theta)} \hat{\mathbf{p}}-\frac{\omega}{c_{2}\left(\Theta^{\prime}\right)} \hat{\mathbf{s}}\right|^{2} \\
& =\frac{\omega^{2}}{c_{1}^{2}(\Theta)}+\frac{\omega^{2}}{c_{2}^{2}\left(\Theta^{\prime}\right)}-\frac{2 \omega^{2}}{c_{1}(\Theta) c_{2}\left(\Theta^{\prime}\right)}(\hat{\mathbf{p}} \cdot \hat{\mathbf{s}}) .
\end{aligned}
$$

The form of the eighth-rank tensor, $\Xi_{\alpha \beta \gamma \delta}^{i j k l}$, is now discussed with regard to cubic crystallites.

\section{A. Statistics of textured cubic polycrystalline media with aligned [001] axis}

The average medium is characterized by the average elastic modulus tensor, $\left\langle C_{i j k l}\right\rangle$. For a statistically transversely isotropic medium, the average modulus is a fourthrank tensor which is a function of the unit vector $\hat{\mathbf{n}}$. The most general form for this tensor is

$$
\begin{aligned}
\left\langle C_{i j k l}(\mathbf{x})\right\rangle \equiv & C_{i j k l}^{0} \\
= & \lambda_{\perp} \delta_{i j} \delta_{k l}+\mu_{\perp}\left(\delta_{i k} \delta_{j l}+\delta_{i l} \delta_{j k}\right) \\
& +A\left(\delta_{i j} \hat{n}_{k} \hat{n}_{l}+\delta_{k l} \hat{n}_{i} \hat{n}_{j}\right) \\
& +B\left(\delta_{i k} \hat{n}_{j} \hat{n}_{l}+\delta_{i l} \hat{n}_{j} \hat{n}_{k}+\delta_{j k} \hat{n}_{i} \hat{n}_{l}+\delta_{j l} \hat{n}_{i} \hat{n}_{k}\right) \\
& +D \hat{n}_{i} \hat{n}_{j} \hat{n}_{k} \hat{n}_{l} .
\end{aligned}
$$

The elastic modulus tensor for a single cubic crystal is given by

$C_{i j k l}(\mathbf{x})=c_{12} \delta_{i j} \delta_{k l}+c_{44}\left(\delta_{i k} \delta_{j l}+\delta_{i l} \delta_{j k}\right)+\eta \sum_{n=1}^{3} a_{i}^{n} a_{j}^{n} a_{k}^{n} a_{l}^{n}$,

where $\eta=c_{11}-c_{12}-2 c_{44}$ is the single-crystal anisotropy. The elements $a_{i}^{n}$ define the rotation matrix in terms of the Euler angles. Each grain is assumed to have a different orientation such that the last term in Eq. (39) is dependent upon $\mathbf{x}$. The ensemble average for this medium is defined by rotation about the aligned axis of the grains. This average is given by

$$
\langle f\rangle=\frac{1}{2 \pi} \int_{0}^{2 \pi} f(\phi) d \phi,
$$

where the dummy variable $\phi$ is defined in the plane perpendicular to the direction of the aligned axis.

Carrying out the average for Eq. (39) and equating like terms with Eq. (38) gives

$$
\begin{gathered}
\lambda_{\perp}=c_{12}+\frac{\eta}{4}, \quad \mu_{\perp}=c_{44}+\frac{\eta}{4}, \\
A=B=-\frac{\eta}{4}, \quad D=\frac{7 \eta}{4} .
\end{gathered}
$$

The average modulus tensor is then

$$
\begin{aligned}
\left\langle C_{i j k l}\right\rangle= & \left(c_{12}+\frac{\eta}{4}\right) \delta_{i j} \delta_{k l}+\left(c_{44}+\frac{\eta}{4}\right)\left(\delta_{i k} \delta_{j l}+\delta_{i l} \delta_{j k}\right) \\
& -\frac{\eta}{4}\left(\delta_{i j} \hat{n}_{k} \hat{n}_{l}+\delta_{k l} \hat{n}_{i} \hat{n}_{j}\right)-\frac{\eta}{4}\left(\delta_{i k} \hat{n}_{j} \hat{n}_{l}+\delta_{i l} \hat{n}_{j} \hat{n}_{k}\right. \\
& \left.+\delta_{j k} \hat{n}_{i} \hat{n}_{l}+\delta_{j l} \hat{n}_{i} \hat{n}_{k}\right)+\frac{7 \eta}{4} \hat{n}_{i} \hat{n}_{j} \hat{n}_{k} \hat{n}_{l} .
\end{aligned}
$$

The eighth-rank covariance, $\Xi_{\alpha \beta \gamma \delta}^{i j k l}$, is also a function of the single vector $\hat{\mathbf{n}}$. It can therefore be constructed of Kronecker deltas and pairs of $\hat{\mathbf{n}}$ 's. The symmetry of the cubic crystal also implies that this tensor must be invariant to permutation of Latin or Greek indices and exchange of all Latin for all Greek indices. Thus, the covariance of moduli fluctuations may be written in terms of 14 independent tensors as

$$
\begin{aligned}
\Xi_{\alpha \beta \gamma \delta \delta}^{i j k l} & =\left\langle C_{i j k l} C_{\alpha \beta \gamma \delta}\right\rangle-\left\langle C_{i j k l}\right\rangle\left\langle C_{\alpha \beta \gamma \delta}\right\rangle \\
& =\eta^{2}\left\langle\left(\sum_{n=1}^{3} a_{i}^{n} a_{j}^{n} a_{k}^{n} a_{l}^{n}\right)\left(\sum_{n=1}^{3} a_{\alpha}^{n} a_{\beta}^{n} a_{\gamma}^{n} a_{\delta}^{n}\right)\right\rangle-\eta^{2}\left\langle\sum_{n=1}^{3} a_{i}^{n} a_{j}^{n} a_{k}^{n} a_{l}^{n}\right)\left\langle\sum_{n=1}^{3} a_{\alpha}^{n} a_{\beta}^{n} a_{\gamma}^{n} a_{\delta}^{n}\right\rangle
\end{aligned}
$$




$$
\begin{aligned}
= & b_{0}\left(\delta_{i j} \delta_{k l}+\delta_{i k} \delta_{j l}+\delta_{i l} \delta_{j k}\right)\left(\delta_{\alpha \beta} \delta_{\gamma \delta}+\delta_{\alpha \gamma} \delta_{\beta \delta}+\delta_{\alpha \delta} \delta_{\beta \gamma}\right)+d_{0}\left(\delta_{L L} \delta_{G G} \delta_{G L} \delta_{G L}-72 \text { terms }\right) \\
& +h_{0}\left(\delta_{G L} \delta_{G L} \delta_{G L} \delta_{G L}-24 \text { terms }\right)+b_{2}\left(\delta_{L L} \delta_{L L} \delta_{G G} \hat{n}_{G} \hat{n}_{G}+\delta_{G G} \delta_{G G} \delta_{L L} \hat{n}_{L} \hat{n}_{L}-36 \text { terms }\right)+c_{2}\left(\delta_{G L} \delta_{G L} \delta_{L L} \hat{n}_{G} \hat{n}_{G}\right. \\
& \left.+\delta_{G L} \delta_{G L} \delta_{G G} \hat{n}_{L} \hat{n}_{L}-144 \text { terms }\right)+d_{2}\left(\hat{n}_{G} \hat{n}_{L} \delta_{G L} \delta_{G G} \delta_{L L}-144 \text { terms }\right)+h_{2}\left(\hat{n}_{G} \hat{n}_{L} \delta_{G L} \delta_{G L} \delta_{G L}-96 \text { terms }\right) \\
& +b_{4}\left[\hat{n}_{i} \hat{n}_{j} \hat{n}_{k} \hat{n}_{l}\left(\delta_{\alpha \beta} \delta_{\gamma \delta}+\delta_{\alpha \gamma} \delta_{\beta \delta}+\delta_{\alpha \delta} \delta_{\beta \gamma}\right)+\hat{n}_{\alpha} \hat{n}_{\beta} \hat{n}_{\gamma} \hat{n}_{\delta}\left(\delta_{i j} \delta_{k l}+\delta_{i k} \delta_{j l}+\delta_{i l} \delta_{j k}\right)\right] \\
& +c_{4}\left(\delta_{G G} \delta_{L L} \hat{n}_{G} \hat{n}_{G} \hat{n}_{L} \hat{n}_{L}-36 \text { terms }\right)+d_{4}\left(\delta_{L L} \delta_{G L} \hat{n}_{L} \hat{n}_{G} \hat{n}_{G} \hat{n}_{G}+\delta_{G G} \delta_{G L} \hat{n}_{L} \hat{n}_{L} \hat{n}_{L} \hat{n}_{G}-96 \text { terms }\right) \\
& +h_{4}\left(\delta_{G L} \delta_{G L} \hat{n}_{L} \hat{n}_{L} \hat{n}_{G} \hat{n} G-72 \text { terms }\right)+b_{6}\left(\delta_{G G} \hat{n}_{G} \hat{n}_{G} \hat{n}_{L} \hat{n}_{L} \hat{n}_{L} \hat{n}_{L}+\delta_{L L} \hat{n}_{L} \hat{n}_{L} \hat{n}_{G} \hat{n}_{G} \hat{n}_{G} \hat{n}_{G}-12 \text { terms }\right) \\
& +d_{6}\left(\delta_{L G} \hat{n}_{L} \hat{n}_{L} \hat{n}_{L} \hat{n}_{G} \hat{n}_{G} \hat{n}_{G}-16 \text { terms }\right)+d_{8} \hat{n}_{i} \hat{n}_{j} \hat{n}_{k} \hat{n}_{l} \hat{n}_{\alpha} \hat{n}_{\beta} \hat{n}_{\gamma} \hat{n}_{\delta} .
\end{aligned}
$$

The above terms not explicitly given are written in terms of $G$ and $L$. This notation implies the form of the terms given by Greek or Latin indices. All possible permutations of the particular form are implied and the number of terms of each form is also given. For example, the factor $c_{4}$ contains terms such as $\delta_{\alpha \beta} \delta_{i j} \hat{n}_{\gamma} \hat{n}_{\delta} \hat{n}_{k} \hat{n}_{l}$ and $\delta_{\alpha \gamma} \delta_{i k} \hat{n}_{\beta} \hat{n}_{\delta} \hat{n}_{j} \hat{n}_{l}$ plus all other permutations of Greek and Latin indices for a total of 36 terms.

The 764 terms in Eq. (43) completely define the covariance in a coordinate-free manner. The averages given in terms of the Euler angles can be carried out and compared with the results of the covariance given by the general form. The coefficients are found to be

$$
\begin{aligned}
& d_{8}=b_{6}=-d_{6}=\frac{9 \eta^{2}}{288}, \\
& b_{4}=-d_{4}=-h_{2}=h_{0}=\frac{3 \eta^{2}}{288}, \\
& d_{2}=c_{2}=b_{0}=-d_{0}=-b_{2}=-c_{4}=\frac{\eta^{2}}{288}, \\
& h_{4}=\frac{5 \eta^{2}}{288} .
\end{aligned}
$$

The forms of the attenuations given in Eqs. (32)-(34) above require various inner products on the covariance ten-

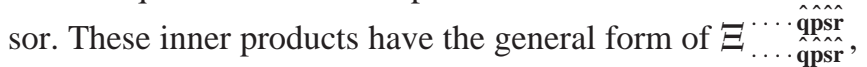
where the vectors $\hat{\mathbf{p}}$ and $\hat{\mathbf{s}}$ represent the incoming and outgoing and propagation directions, respectively, and the vectors $\hat{\mathbf{q}}$ and $\hat{\mathbf{r}}$ are vectors defining the polarization directions of the particular wave. These vectors are perpendicular to the plane defined by $\hat{\mathbf{s}}$ or $\hat{\mathbf{p}}$ and $\hat{\mathbf{n}}$ (for SH waves) or they lie in this plane (for $\mathrm{qP}$ and $\mathrm{qSV}$ ). This general inner product can be written in terms of various combinations of inner products of the vectors involved. The most general form is given explicitly elsewhere. ${ }^{16}$
Specific inner products required for the calculation of the attenuations are now determined. The attenuations will vary angularly only within the plane defined by the propagation direction and the fiber direction. Therefore, without loss of generality, a reference plane is defined as the $\hat{\mathbf{p}}-\hat{\mathbf{n}}$ plane. The following vectors are then defined with respect to a general $x y z$ coordinate system as

$$
\begin{aligned}
& \hat{\mathbf{n}}=\hat{\mathbf{z}}, \\
& \hat{\mathbf{p}}=\hat{\mathbf{y}} \sin \Theta+\hat{\mathbf{z}} \cos \Theta, \\
& \hat{\mathbf{s}}=\hat{\mathbf{x}} \sin \Theta^{\prime} \cos \phi^{\prime}+\hat{\mathbf{y}} \sin \Theta^{\prime} \sin \phi^{\prime}+\hat{\mathbf{z}} \cos \Theta^{\prime} .
\end{aligned}
$$

The polarization vectors are then defined with respect to these angles and $\psi$ [Eq. (24)] as

$$
\begin{aligned}
& \hat{\mathbf{u}}_{1}=\hat{\mathbf{x}}, \\
& \hat{\mathbf{u}}_{2}=\hat{\mathbf{y}} \sin \gamma+\hat{\mathbf{z}} \cos \gamma, \\
& \hat{\mathbf{u}}_{3}=-\hat{\mathbf{y}} \cos \gamma+\hat{\mathbf{z}} \sin \gamma,
\end{aligned}
$$

and

$$
\begin{aligned}
& \hat{\mathbf{v}}_{\mathbf{1}}=\hat{\mathbf{x}} \sin \phi^{\prime}-\hat{\mathbf{y}} \cos \phi^{\prime}, \\
& \hat{\mathbf{v}}_{\mathbf{2}}=\hat{\mathbf{x}} \sin \gamma^{\prime} \cos \phi^{\prime}+\hat{\mathbf{y}} \sin \gamma^{\prime} \sin \phi^{\prime}+\hat{\mathbf{z}} \cos \gamma^{\prime}, \\
& \hat{\mathbf{v}}_{\mathbf{3}}=-\hat{\mathbf{x}} \cos \gamma^{\prime} \cos \phi^{\prime}-\hat{\mathbf{y}} \cos \gamma^{\prime} \sin \phi^{\prime}+\hat{\mathbf{z}} \sin \gamma^{\prime},
\end{aligned}
$$

where the angles $\gamma$ and $\gamma^{\prime}$ used above are defined by

$$
\gamma=\Theta+\psi(\Theta), \quad \gamma^{\prime}=\Theta^{\prime}+\psi\left(\Theta^{\prime}\right) .
$$

These angles, $\gamma$ and $\gamma^{\prime}$, define the orientation angle of the $\mathrm{qP}$ wave with respect to the $\hat{\mathbf{n}}$ direction in the $\hat{\mathbf{p}}-\hat{\mathbf{n}}$ and $\hat{\mathbf{s}}-\hat{\mathbf{n}}$ planes, respectively.

Using these definitions of the relevant unit vectors, the required inner products simplify considerably. The inner products are for $\alpha_{\mathrm{SH}}$, 


$$
\begin{aligned}
& \underset{\cdots \cdots \hat{\mathbf{u}}_{\hat{\mathbf{p}} \hat{\mathbf{s}} \hat{\mathbf{v}}_{1}}}{\Xi \cdots \hat{\mathbf{u}}_{\mathbf{1}} \mathbf{\mathbf { p }} \mathbf{\mathbf { v }} \mathbf{1}}=\frac{\eta^{2}}{32} \sin ^{2} \Theta^{\prime} \sin ^{2} \Theta, \\
& \Xi_{\cdots \cdot \hat{\mathbf{u}}_{1} \hat{\mathbf{p}} \hat{\hat{\hat{p}}} \hat{\mathbf{s}}_{2} \hat{\mathbf{v}}_{2}}=\frac{\eta^{2}}{32} \sin ^{2} \Theta^{\prime} \sin ^{2} \gamma^{\prime} \sin ^{2} \Theta, \\
& \Xi_{\cdots \hat{\mathbf{u}}_{1} \hat{\mathbf{p}} \hat{\mathbf{p}} \hat{\mathbf{v}}_{3} \mathbf{v}_{3}}=\frac{\eta^{2}}{32} \sin ^{2} \Theta^{\prime} \cos ^{2} \gamma^{\prime} \sin ^{2} \Theta,
\end{aligned}
$$

$$
\Xi_{\cdots \cdot \hat{\mathbf{u}}_{2} \hat{\hat{p}} \hat{\hat{\hat{p}}} \hat{\mathbf{s}}_{3} \mathbf{v}_{3}}=\frac{\eta^{2}}{32} \sin ^{2} \Theta^{\prime} \cos ^{2} \gamma^{\prime} \sin ^{2} \gamma \sin ^{2} \Theta
$$

$$
\Xi_{\cdots \cdots \hat{\mathbf{u}}_{3} \hat{p} \hat{\mathbf{p}} \hat{\mathbf{s}} \hat{\mathbf{v}}_{3} \hat{\mathbf{v}}_{3}}=\frac{\eta^{2}}{32} \sin ^{2} \Theta^{\prime} \cos ^{2} \gamma^{\prime} \cos ^{2} \gamma \sin ^{2} \Theta
$$

where $\gamma$ and $\gamma^{\prime}$ are defined in Eq. (48). The expressions given by Eqs. (49)-(51) are also directly related to the diffuse propagation including backscatter. ${ }^{11-13}$ to

Using the above inner products, the attenuations reduce

$$
\begin{aligned}
\alpha_{\mathrm{SH}}(\Theta)= & \frac{\omega^{4} \sin ^{2} \Theta}{c_{\mathrm{SH}}^{3}(\Theta)} \frac{\eta^{2} \pi}{128} \int_{0}^{2 \pi} d \phi^{\prime} \int_{0}^{\pi}\left[\frac{\widetilde{W}_{\mathrm{SH}-\mathrm{SH}}(\hat{\mathbf{p}}, \hat{\mathbf{s}}) \sin ^{3} \Theta^{\prime}}{c_{\mathrm{SH}}^{5}\left(\Theta^{\prime}\right)}\right. \\
& +\frac{\widetilde{W}_{\mathrm{SH}-\mathrm{qP}}(\hat{\mathbf{p}}, \hat{\mathbf{s}}) \sin ^{3} \Theta^{\prime} \sin ^{2} \gamma^{\prime}}{c_{\mathrm{qP}}^{5}\left(\Theta^{\prime}\right)} \\
& \left.+\frac{\widetilde{W}_{\mathrm{SH}-\mathrm{qSV}}(\hat{\mathbf{p}}, \hat{\mathbf{s}}) \sin ^{3} \Theta^{\prime} \cos ^{2} \gamma^{\prime}}{c_{\mathrm{qSV}}^{5}\left(\Theta^{\prime}\right)}\right] d \Theta^{\prime}
\end{aligned}
$$

$$
\begin{aligned}
& \alpha_{\mathrm{qP}}(\Theta)=\frac{\omega^{4} \sin ^{2} \gamma \sin ^{2} \Theta}{c_{\mathrm{qP}}^{3}(\Theta)} \frac{\eta^{2} \pi}{128} \int_{0}^{2 \pi} d \phi^{\prime} \\
& \times \int_{0}^{\pi}\left[\frac{\widetilde{W}_{\mathrm{qP}-\mathrm{SH}}(\hat{\mathbf{p}}, \hat{\mathbf{s}}) \sin ^{3} \Theta^{\prime}}{c_{\mathrm{SH}}^{5}\left(\Theta^{\prime}\right)}\right. \\
& +\frac{\widetilde{W}_{\mathrm{qP}-\mathrm{qP}}(\hat{\mathbf{p}}, \hat{\mathbf{s}}) \sin ^{3} \Theta^{\prime} \sin ^{2} \gamma^{\prime}}{c_{\mathrm{qP}}^{5}\left(\Theta^{\prime}\right)} \\
& \left.+\frac{\widetilde{W}_{\mathrm{qP}-\mathrm{qSV}}(\hat{\mathbf{p}}, \hat{\mathbf{s}}) \sin ^{3} \Theta^{\prime} \cos ^{2} \gamma^{\prime}}{c_{\mathrm{qSV}}^{5}\left(\Theta^{\prime}\right)}\right] d \Theta^{\prime}, \\
& \alpha_{\mathrm{qSV}}(\Theta)=\frac{\omega^{4} \cos ^{2} \gamma \sin ^{2} \Theta}{c_{\mathrm{qSV}}^{3}(\Theta)} \frac{\eta^{2} \pi}{128} \int_{0}^{2 \pi} d \phi^{\prime} \\
& \times \int_{0}^{\pi}\left[\frac{\widetilde{W}_{\mathrm{qSV}-\mathrm{SH}}(\hat{\mathbf{p}}, \hat{\mathbf{s}}) \sin ^{3} \Theta^{\prime}}{c_{\mathrm{SH}}^{5}\left(\Theta^{\prime}\right)}\right. \\
& +\frac{\widetilde{W}_{\mathrm{qSV}-\mathrm{qP}}(\hat{\mathbf{p}}, \hat{\mathbf{s}}) \sin ^{3} \Theta^{\prime} \sin ^{2} \gamma^{\prime}}{c_{\mathrm{qP}}^{5}\left(\Theta^{\prime}\right)} \\
& \left.+\frac{\widetilde{W}_{\mathrm{qSV}-\mathrm{qSV}}(\hat{\mathbf{p}}, \hat{\mathbf{s}}) \sin ^{3} \Theta^{\prime} \cos ^{2} \gamma^{\prime}}{c_{\mathrm{qSV}}^{5}\left(\Theta^{\prime}\right)}\right] d \Theta^{\prime} .
\end{aligned}
$$

These expressions are now nondimensionalized and simplified. Reference wave speeds are needed for the nondimensionalization. Rather than using the Voigt average wave speeds, wave speeds characteristic of the anisotropic medium are used. Average wave speeds are defined as

$$
\bar{c}_{\beta}=\frac{1}{2} \int_{0}^{\pi} c_{\beta}(\Theta) \sin \Theta d \Theta,
$$

for each wave type, $\beta$. The three nondimensional frequencies are then defined as $x_{\beta}=\omega L / \bar{c}_{\beta}$. Finally, dimensionless quantities which describe the slowness surface for each wave type are defined by $r_{\beta}(\Theta)=\bar{c}_{\beta} / c_{\beta}(\Theta)$.

The expressions for the spatial correlation functions must now be discussed. Using the form of the spatial Fourier transform of the correlation function, given by Eq. (36), the functions $\widetilde{W}_{\beta-\gamma}(\hat{\mathbf{p}}, \hat{\mathbf{s}})$ are determined. In terms of the above dimensionless quantities, we find

$$
\widetilde{W}_{\beta-\gamma}(\hat{\mathbf{p}}, \hat{\mathbf{s}})=\frac{L^{3}}{\pi^{2}\left(1+x_{\beta}^{2} r_{\beta}^{2}(\Theta)+x_{\gamma}^{2} r_{\gamma}^{2}\left(\Theta^{\prime}\right)-2 x_{\beta} x_{\gamma} r_{\beta}(\Theta) r_{\gamma}\left(\Theta^{\prime}\right) \hat{\mathbf{p}} \cdot \hat{\mathbf{s}}\right)^{2}}
$$

for the incoming wave type $\beta$ and outgoing wave type $\gamma$. The inner product, $\hat{\mathbf{p}} \cdot \hat{\mathbf{s}}=\cos \Theta \cos \Theta^{\prime}+\sin \Theta \sin \Theta^{\prime} \sin \phi^{\prime}$. The integration over $\phi^{\prime}$ in Eqs. (52)-(54) can be done in closed form. The resulting dimensionless attenuations are then 


$$
\begin{aligned}
\alpha_{\mathrm{SH}}(\Theta) L= & x_{\mathrm{SH}}^{4} \frac{\eta^{2}}{64 \rho^{2} \bar{c}_{\mathrm{SH}}^{4}} r_{\mathrm{SH}}^{3}(\Theta) \sin ^{2} \Theta \\
& \times\left[I_{\mathrm{SH}-\mathrm{SH}}+I_{\mathrm{SH}-\mathrm{qP}}\left(\frac{\bar{c}_{\mathrm{SH}}}{\bar{c}_{\mathrm{qP}}}\right)^{5}+I_{\mathrm{SH}-\mathrm{qSV}}\left(\frac{\bar{c}_{\mathrm{SH}}}{\bar{c}_{\mathrm{qSV}}}\right)^{5}\right],
\end{aligned}
$$

$$
\begin{aligned}
\alpha_{\mathrm{qP}}(\Theta) L= & x_{\mathrm{qP}}^{4} \frac{\eta^{2}}{64 \rho^{2} \bar{c}_{\mathrm{qP}}^{4}} r_{\mathrm{qP}}^{3}(\Theta) \sin ^{2} \Theta \sin ^{2} \gamma \\
& \times\left[I_{\mathrm{qP}-\mathrm{SH}}\left(\frac{\bar{c}_{\mathrm{qP}}}{\bar{c}_{\mathrm{SH}}}\right)^{5}+I_{\mathrm{qP}-\mathrm{qP}}+I_{\mathrm{qP}-\mathrm{qSV}}\left(\frac{\bar{c}_{\mathrm{qP}}}{\bar{c}_{\mathrm{qSV}}}\right)^{5}\right],
\end{aligned}
$$

$$
\begin{aligned}
\alpha_{\mathrm{qSV}}(\Theta) L= & x_{\mathrm{qSV}}^{4} \frac{\eta^{2}}{64 \rho^{2} \bar{c}_{\mathrm{qSV}}^{4}} r_{\mathrm{qSV}}^{3}(\Theta) \sin ^{2} \Theta \cos ^{2} \gamma \\
& \times\left[I_{\mathrm{qSV}-\mathrm{SH}}\left(\frac{\bar{c}_{\mathrm{qSV}}}{\bar{c}_{\mathrm{SH}}}\right)^{5}+I_{\mathrm{qSV}-\mathrm{qP}}\left(\frac{\bar{c}_{\mathrm{qSV}}}{\bar{c}_{\mathrm{qP}}}\right)^{5}\right. \\
& \left.+I_{\mathrm{qSV}-\mathrm{qSV}}\right]
\end{aligned}
$$

with the density, $\rho$, now included.

The above terms within the square brackets represent integrals which are given by the general forms

$$
\begin{aligned}
& I_{\beta-\mathrm{SH}}=\int_{0}^{\pi} \frac{r_{\mathrm{SH}}^{5}\left(\Theta^{\prime}\right) X_{\beta-\mathrm{SH}} \sin ^{3} \Theta^{\prime}}{\left(X_{\beta-\mathrm{SH}}^{2}-Y_{\beta-\mathrm{SH}}^{2}\right)^{3 / 2}} d \Theta^{\prime}, \\
& I_{\beta-\mathrm{qP}}=\int_{0}^{\pi r_{\mathrm{qP}}^{5}\left(\Theta^{\prime}\right) X_{\beta-\mathrm{q}} \sin ^{2}\left(\Theta^{\prime}+\psi\left(\Theta^{\prime}\right)\right) \sin ^{3} \Theta^{\prime}} \frac{\left(X_{\beta-\mathrm{qP}}^{2}-Y_{\beta-\mathrm{qP}}^{2}\right)^{3 / 2}}{\Theta^{\prime},}
\end{aligned}
$$

$I_{\beta-\mathrm{qSV}}$

$$
=\int_{0}^{\pi} \frac{r_{\mathrm{qSV}}^{5}\left(\Theta^{\prime}\right) X_{\beta-\mathrm{qSV}} \cos ^{2}\left(\Theta^{\prime}+\psi\left(\Theta^{\prime}\right)\right) \sin ^{3} \Theta^{\prime}}{\left(X_{\beta-\mathrm{qSV}}^{2}-Y_{\beta-\mathrm{qSV}}^{2}\right)^{3 / 2}} d \Theta^{\prime},
$$

with

$$
\begin{aligned}
X_{\beta-\gamma}= & 1+x_{\beta}^{2} r_{\beta}^{2}(\Theta)+x_{\gamma}^{2} r_{\gamma}^{2}\left(\Theta^{\prime}\right) \\
& -2 x_{\beta} x_{\gamma} r_{\beta}(\Theta) r_{\gamma}\left(\Theta^{\prime}\right) \cos \Theta \cos \Theta^{\prime}, \\
Y_{\beta-\gamma}= & 2 x_{\beta} x_{\gamma} r_{\beta}(\Theta) r_{\gamma}\left(\Theta^{\prime}\right) \sin \Theta \sin \Theta^{\prime},
\end{aligned}
$$

for the different wave types, $\beta$ and $\gamma$.

In the Rayleigh limit, these integrals simplify considerably. They become independent of incident direction and frequency and are given by

$$
\begin{aligned}
& I_{\beta-\mathrm{SH}}=\int_{0}^{\pi} r_{\mathrm{SH}}^{5}\left(\Theta^{\prime}\right) \sin ^{3} \Theta^{\prime} d \Theta^{\prime}, \\
& I_{\beta-\mathrm{qP}}=\int_{0}^{\pi} r_{\mathrm{qP}}^{5}\left(\Theta^{\prime}\right) \sin ^{2}\left(\Theta^{\prime}+\psi\left(\Theta^{\prime}\right)\right) \sin ^{3} \Theta^{\prime} d \Theta^{\prime},
\end{aligned}
$$

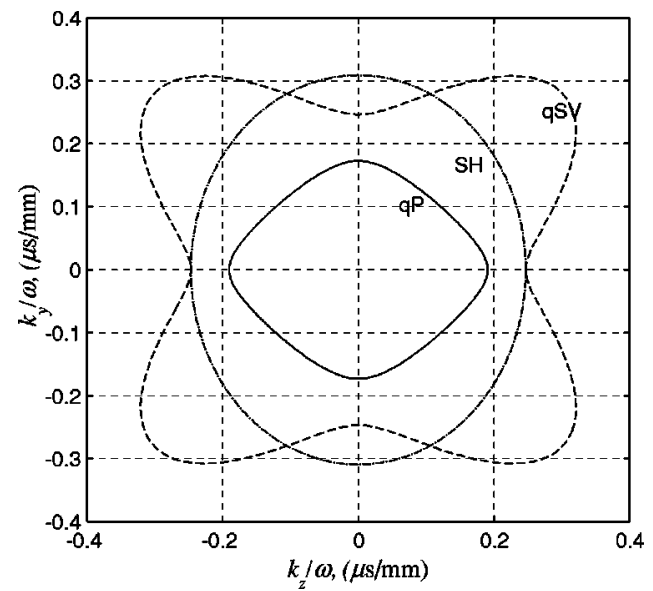

FIG. 1. Slowness surfaces for stainless steel with texture.

$$
I_{\beta-\mathrm{qSV}}=\int_{0}^{\pi} r_{\mathrm{qSV}}^{5}\left(\Theta^{\prime}\right) \cos ^{2}\left(\Theta^{\prime}+\psi\left(\Theta^{\prime}\right)\right) \sin ^{3} \Theta^{\prime} d \Theta^{\prime},
$$

for all incoming wave types $\beta$. Thus, the angular dependence of the attenuations in the Rayleigh limit is seen explicitly. The attenuations are dependent upon the cube of the slowness multiplied by a factor related to the polarization type. The attenuation $\alpha_{\mathrm{SH}}$ varies as $r_{\mathrm{SH}}^{3}(\Theta) \sin ^{2} \Theta, \alpha_{\mathrm{qP}}$ varies as $r_{\mathrm{qP}}^{3}(\Theta) \sin ^{2}(\Theta+\psi(\Theta)) \sin ^{2} \Theta$, and $\alpha_{\mathrm{qSV}}$ varies as $r_{\mathrm{qSV}}^{3}(\Theta) \cos ^{2}(\Theta+\psi(\Theta)) \sin ^{2} \Theta$.

\section{B. Results for stainless steel}

Numerical results are now presented for the specific case of stainless steel with aligned [001] axes. The material properties used are $^{5}$

$$
\begin{aligned}
& c_{11}=2.16 \times 10^{11} \mathrm{~Pa}, \quad c_{12}=1.45 \times 10^{11} \mathrm{~Pa}, \\
& c_{44}=1.29 \times 10^{11} \mathrm{~Pa}, \quad \rho=7860 \mathrm{~kg} / \mathrm{m}^{3} .
\end{aligned}
$$

The slowness surfaces calculated using the dispersion relations for the bare medium, Eqs. (26), are shown in Fig. 1. These results agree well with those of previous authors. ${ }^{5}$

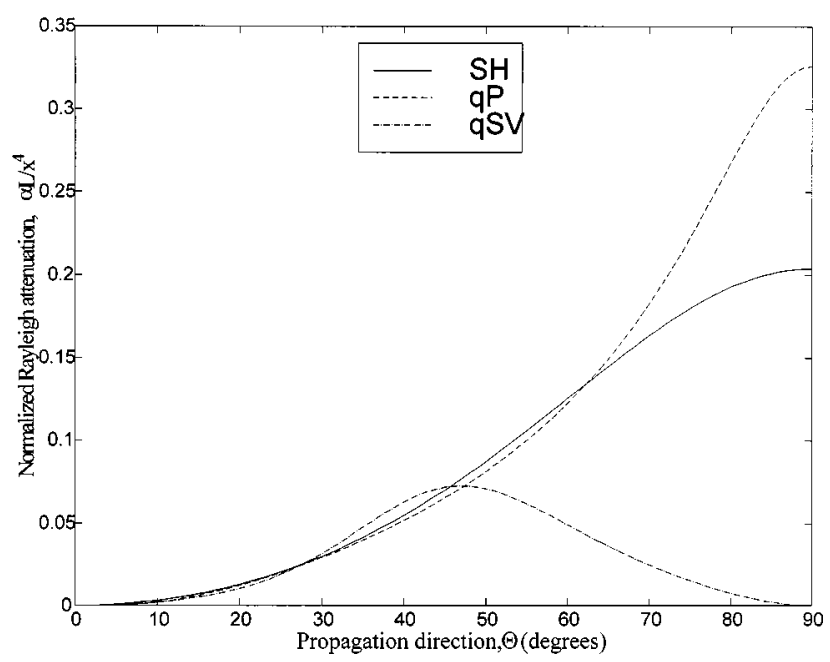

FIG. 2. Dimensionless attenuation in the Rayleigh limit as a function of direction for the SH, qP, and qSV waves. The dimensionless attenuation $\alpha L$ has been normalized by the fourth power of the dimensionless frequency for the respective wave type: $\alpha_{\mathrm{SH}} L / x_{\mathrm{SH}}^{4}, \alpha_{\mathrm{qP}} L / x_{\mathrm{qP}}^{4}$, and $\alpha_{\mathrm{qSV}} L / x_{\mathrm{qSV}}^{4}$. 


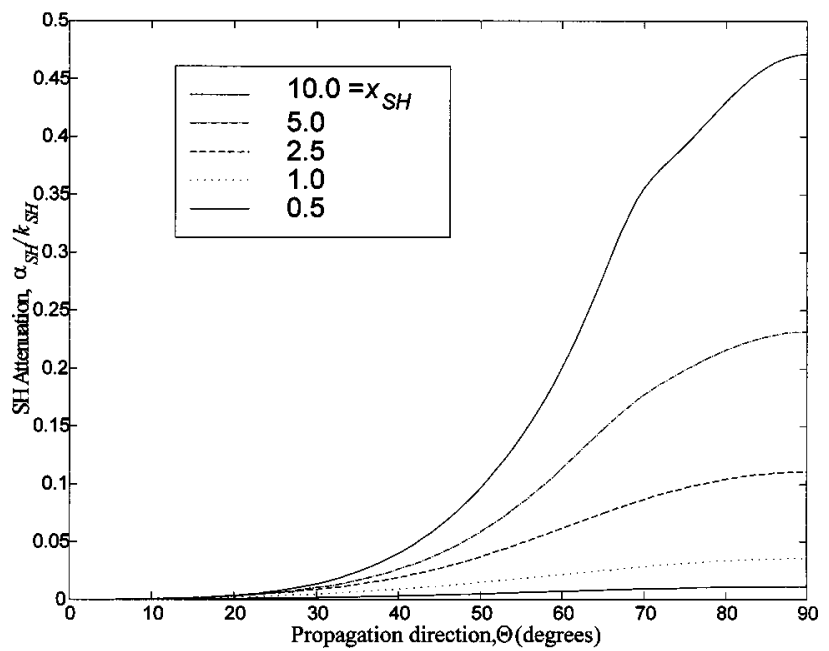

FIG. 3. Angular dependence of the normalized SH attenuation, $\alpha_{\mathrm{SH}} / k_{\mathrm{SH}}$, for various frequencies, $x_{\mathrm{SH}}$.

Attenuation results, using Eqs. (57)-(59) are given in terms of the single dimensionless frequency $x_{\mathrm{SH}}$ $=\omega L / \bar{c}_{\mathrm{SH}}$. The calculations are primarily limited to $x_{\mathrm{SH}}$ $<10$ corresponding to the onset of the geometric optics limit observed by Ahmed and Thompson. ${ }^{5}$ (Their dimensionless frequency, $\chi=k d$, is based on the grain diameter. Here, this quantity contains the grain radius.) The required integrals were calculated numerically using a Newton-Cotes quadrature function quad8 available in the software package Matlab. ${ }^{17}$

In the Rayleigh limit, the integrals in Eqs. (60) reduce to those given by Eqs. (62). For the case considered here, with parameters given by Eq. (63), these integrals are $I_{\beta-\mathrm{SH}}$ $=1.623, I_{\beta-\mathrm{qP}}=1.068$, and $I_{\beta-\mathrm{qSV}}=0.6271$. In the Rayleigh regime the attenuation depends on the fourth power of frequency. Thus, the angular dependence of the three attenuations is described by the quantity $\alpha L / x^{4}$. This parameter for each wave type is shown in Fig. 2. The $S H$ and qP waves have their maxima perpendicular to the fiber direction. All wave types have zero attenuation in the fiber direction-the material properties do not vary in that direction. The qSV wave is seen to have zero attenuation in the fiber direction and perpendicular to the fiber direction. This result is the same as previous work. ${ }^{4,5}$ Here, however, the same symmetry is not seen. The qSV attenuation actually peaks at $47.1^{\circ}$ rather than $45^{\circ}$. The qSV attenuation greater than the peak is slightly higher than that for propagation directions below the peak as well. Thus, the inclusion of the wave polarizations through the anisotropic Green's dyadic results in slight differences from the case without.

Results outside the Rayleigh regime are calculated using the complete integrals, Eqs. (60). The directional dependence of the attenuation as a function of frequency is presented first. Figure 3 shows the normalized $\mathrm{SH}$ attenuation, $\alpha_{\mathrm{SH}} / k_{\mathrm{SH}}$, as a function of propagation direction for five different frequencies. These results may be contrasted with the results in the Rayleigh limit. As the frequency increases we see that the attenuation in the cross-fiber direction increases more than in other directions. The results for the normalized $\mathrm{qP}$ attenuation, $\alpha_{\mathrm{qP}} / k_{\mathrm{qP}}$, are shown in Fig. 4. These results

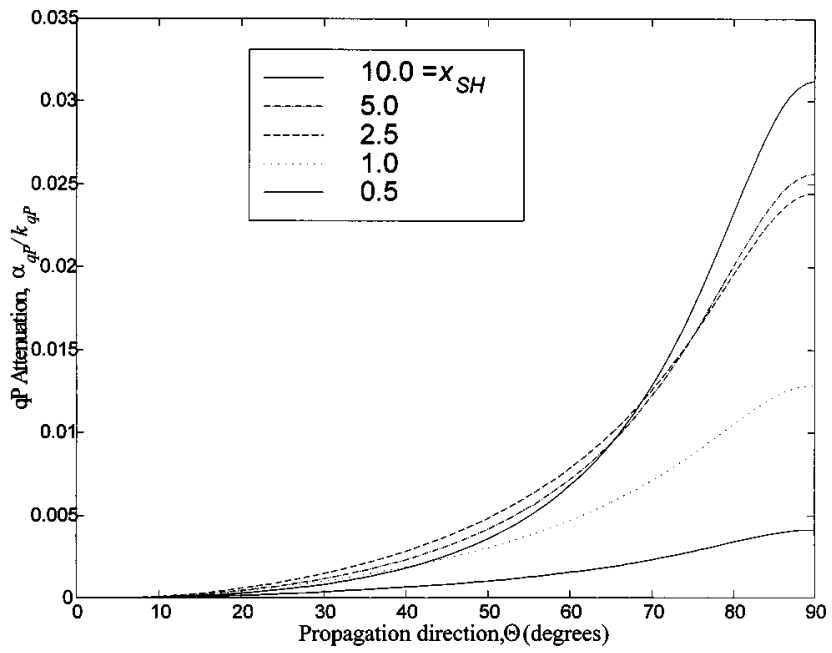

FIG. 4. Angular dependence of the normalized qP attenuation, $\alpha_{\mathrm{qP}} / k_{\mathrm{qP}}$, for various frequencies, $x_{\mathrm{SH}}$.

are similar to the SH attenuation. The cross-fiber attenuation increases more rapidly than other directions as the frequency increases. Similar results were seen by Hirsekorn ${ }^{4}$ and Ahmed and Thompson. ${ }^{5}$

The normalized qSV attenuation, $\alpha_{\mathrm{qSV}} / k_{\mathrm{qSV}}$, is shown in Fig. 5 at the same frequencies as Figs. 3 and 4. The attenuation in the fiber and cross-fiber directions is zero as expected from the form of the modulus fluctuations. The direction of maximum $\alpha_{\mathrm{qSV}}$, as observed in Fig. 5, is seen to be a function of frequency. This result is shown more explicitly in Fig. 6 in which the direction of maximum $\alpha_{\mathrm{qSV}}$ is plotted versus $x_{\mathrm{SH}}$. This direction is $47.1^{\circ}$ in the Rayleigh limit as was seen in Fig. 2. The direction of maximum $\alpha_{\mathrm{qSV}}$ then increases with increasing frequency. The peak in this maximum occurs at a frequency of about $x_{\mathrm{SH}}=1.8$ at an angle of $51.8^{\circ}$. The direction of maximum $\alpha_{\mathrm{qSV}}$ then decreases for larger values of $x_{\mathrm{SH}}$. The result presented here differs from that presented by Ahmed and Thompson. ${ }^{5}$ Their maximum $\alpha_{\mathrm{qSV}}$ occurs at $45^{\circ}$ in the Rayleigh regime. At higher frequencies they noted an increase in the angle of maximum $\alpha_{\mathrm{qSV}}$, although the shift from $45^{\circ}$ is not as dra-

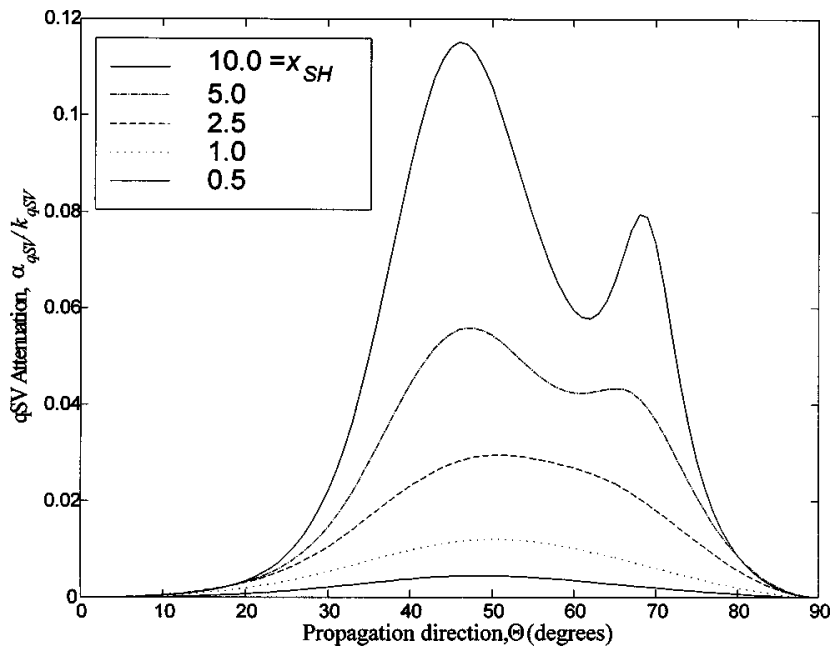

FIG. 5. Angular dependence of the normalized qSV attenuation, $\alpha_{\mathrm{qSV}} / k_{\mathrm{qSV}}$, for various frequencies, $x_{\mathrm{SH}}$. 


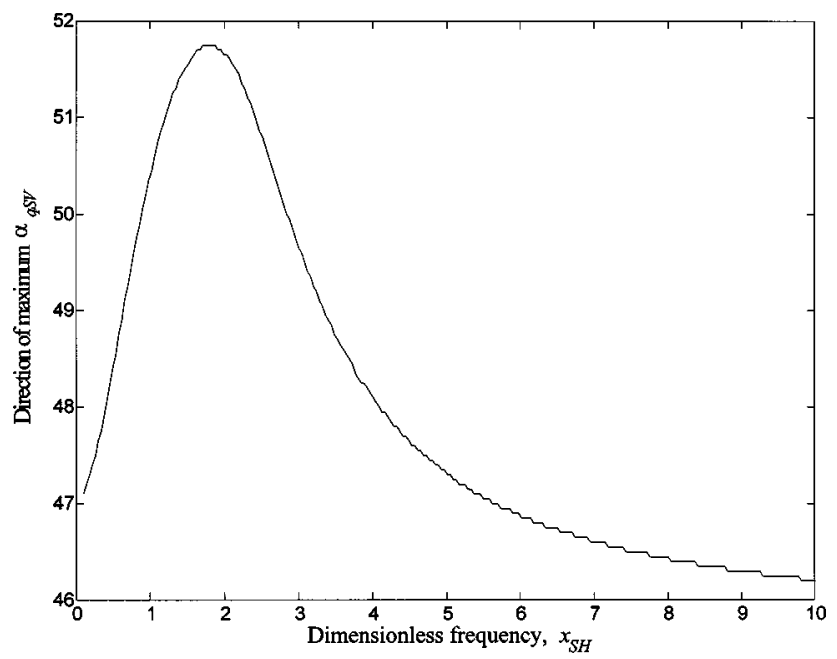

FIG. 6. Direction of maximum qSV attenuation as a function of frequency, $x_{\mathrm{SH}}$. This angle is about $47.1^{\circ}$ in the low-frequency limit, peaks at about $51.8^{\circ}$ at $x_{\mathrm{SH}}=1.8$ and then decreases for higher frequencies.

matic as that seen here. This difference is also attributed to the inclusion of polarization direction in the present results.

The appearance of the additional peak in the SV attenuation at about $69^{\circ}$ is observed. The presence of such a peak at the same angle was also observed by Ahmed and Thompson. They suggested that this phenomenon was related to the stochastic-geometric transition. However, the presence of this peak in the present results shows that this is not the case- the above derivation is limited to frequencies excluding the high-frequency geometric limit. The above forms of the attenuation allow this phenomenon to be examined more closely. The integrals $I_{\mathrm{SH}-\mathrm{qSV}}$ and $I_{\mathrm{qSV}-\mathrm{SH}}$ in the equations for attenuation, Eqs. (60), have peaks at about $69^{\circ}$. This peak is the result of the form of the spatial correlation function between the $\mathrm{SH}$ and qSV modes. The term $X_{\mathrm{SH}-\mathrm{qSV}}\left(X_{\mathrm{SH}-\mathrm{qSV}}^{2}-Y_{\mathrm{SH}-\mathrm{qSV}}^{2}\right)^{-3 / 2}$ determines the specific direction of this peak and is a function of the angular phase velocities and propagation direction. Closer examination shows that this peak occurs at the angle corresponding to the intersection of the SH and qSV slowness surfaces of $69.3^{\circ}$. The appearance of a similar peak is seen in the angular plot of SH attenuation, Fig. 3, at $x_{\mathrm{SH}}=10$.

Finally, results are presented for the normalized attenuations as a function of frequency for several propagation directions. In Figs. 7 and 8 the normalized SH and qP attenuations are plotted versus dimensionless frequency, $x_{\mathrm{SH}}$, for propagation directions of $45^{\circ}, 69.5^{\circ}$, and $90^{\circ}$. The $\mathrm{SH}$ attenuation in the cross-fiber direction is seen to increase more rapidly than other directions for increasing frequency. The $\mathrm{qP}$ attenuation has a local maximum which is a function of propagation direction. Similar results were given by Ahmed and Thompson. ${ }^{5}$ The normalized qSV attenuation is plotted versus frequency, $x_{\mathrm{SH}}$, for propagation directions of $45^{\circ}$ and $69.5^{\circ}$ in Fig. 9. The attenuation at $69.5^{\circ}$ increases more rapidly than that at $45^{\circ}$. However, at higher frequencies the ratio between the two appears to be constant. Thus, the peak at $69.5^{\circ}$ observed in the angular plots, Fig. 5 , is not expected to become larger than that of $45^{\circ}$, within the frequency limits used here.

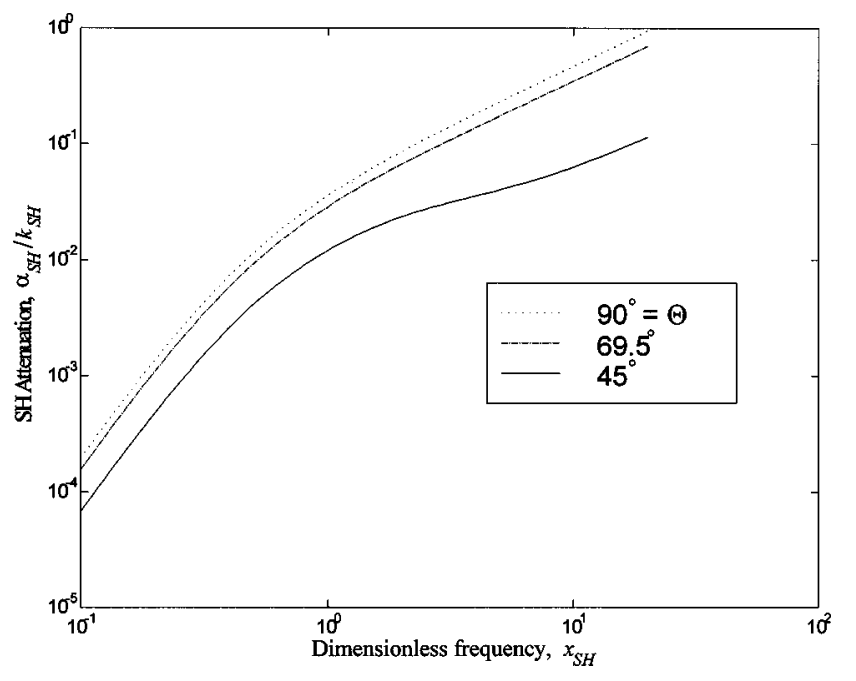

FIG. 7. Normalized $\mathrm{SH}$ attenuation, $\alpha_{\mathrm{SH}} / k_{\mathrm{SH}}$, as a function of dimensionless frequency, $x_{\mathrm{SH}}$, for propagation directions of $45^{\circ}, 69.5^{\circ}$, and $90^{\circ}$.

\section{DISCUSSION}

The propagation and scattering of elastic waves in heterogeneous, anisotropic media has been examined. Appropriate ensemble averaging of the elastic wave equation resulted in the Dyson equation, governing the mean field. The problem was further specified for the case of transverse isotropy. The Green's dyadic for a transversely isotropic medium was used to derive expressions for the attenuation of the shear horizontal, quasicompressional, and quasishear waves. The covariance of moduli fluctuations were determined in coordinate-free form for cubic polycrystalline materials with aligned [001] axes. The final forms of the attenuations for the three wave types were given by simple expressions involving integrations over the unit circle. The integrands are dependent upon inner products on the convariance of modulus fluctuations and factors of phase velocity. The simple form of the results makes them particularly useful for nondestructive testing and materials characterization research and for inclusion of attenuation in numerical models of elastic wave propagation such as those by Spies. ${ }^{14,18,19}$ The results pre-

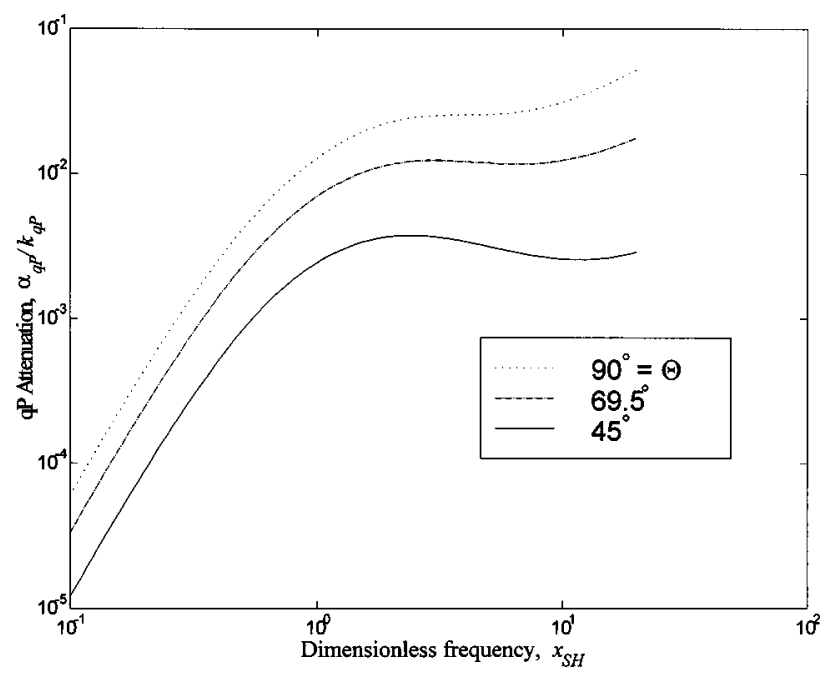

FIG. 8. Normalized qP attenuation, $\alpha_{\mathrm{qP}} / k_{\mathrm{qP}}$, as a function of dimensionless frequency, $x_{\mathrm{SH}}$, for propagation directions of $45^{\circ}, 69.5^{\circ}$, and $90^{\circ}$. 


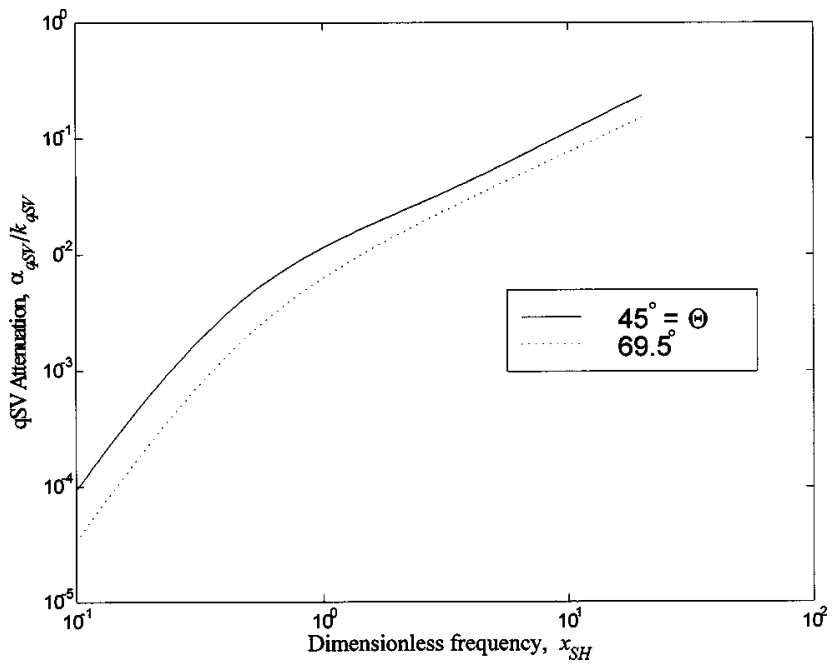

FIG. 9. Normalized qSV attenuation, $\alpha_{\mathrm{qSV}} / k_{\mathrm{qSV}}$, as a function of dimensionless frequency, $x_{\mathrm{SH}}$, for propagation directions of $45^{\circ}$ and $69.5^{\circ}$. The attenuation at $69.5^{\circ}$ is initially much smaller than that at $45^{\circ}$. It then increases more quickly than that at $45^{\circ}$. The ratio between the two appears to then be constant at higher frequencies.

sented here are also directly related to diffuse field methods such as backscatter techniques. In addition, the above formalism holds for other forms of global anisotropy. The bare Green's dyadic may be expanded in a similar fashion. The main difficulty will then lie in the determination of the form of the covariance of elastic moduli fluctuations.

\section{ACKNOWLEDGMENTS}

The Alexander von Humboldt Foundation and the Fraunhofer-Institut für zerstörungsfreie Prüfverfahren (IzfP), in Saarbrücken, Germany are both gratefully acknowledged for support during a portion of this work. The author also thanks M. Spies for conversations regarding this work.

${ }^{1}$ F. E. Stanke and G. S. Kino, " A unified theory for elastic wave propagation in polycrystalline materials," J. Acoust. Soc. Am. 75, 665-681 (1984).
${ }^{2}$ F. C. Karal and J. B Keller, "Elastic, electromagnetic, and other waves in a random medium," J. Math. Phys. 5, 537-547 (1964).

${ }^{3}$ S. Hirsekorn, "The scattering of ultrasonic waves in polycrystalline materials with texture," J. Acoust. Soc. Am. 77, 832-843 (1985).

${ }^{4} \mathrm{~S}$. Hirsekorn, "Directional dependence of ultrasonic propagation in textured polycrystals," J. Acoust. Soc. Am. 79, 1269-1279 (1986).

${ }^{5} \mathrm{~S}$. Ahmed and R. B. Thompson, "Propagation of elastic waves in equiaxed stainless-steel polycrystals with aligned [001] axes," J. Acoust. Soc. Am. 99, 2086-2096 (1996).

${ }^{6} \mathrm{~S}$. Ahmed and R. B. Thompson, in Effects of Preferred Grain Orientation and Grain Elongation on Ultrasonic Wave Propagation in Stainless Steel, edited by D. O. Thompson and D. E. Chimenti (Plenum, New York, 1992), Vol. 11B, pp. 1999-2006.

${ }^{7} \mathrm{~S}$. Ahmed and R. B. Thompson, "Influence of Columnar Microstructure on Ultrasonic Backscattering," in Review of Progress in QNDE, edited by D. O. Thompson and D. E. Chimenti (Plenum, New York, 1995), Vol. 14, pp. 1617-1624.

${ }^{8}$ E. M. Lifshits and G. D. Parkhamovski, Zh. Eksp. Teor. Fiz. 20, 175-182 (1950).

${ }^{9}$ U. Frisch, "Wave Propagation in Random Media," in Probalistic Methods in Applied Mathematics, edited by A. T. Barucha-Reid (Academic, New York, 1968), Vol. 1, pp. 75-198.

${ }^{10}$ R. L. Weaver, "Diffusion of Ultrasound in Polycrystals," J. Mech. Phys. Solids 38, 55-86 (1990).

${ }^{11}$ J. A. Turner and R. L. Weaver, "Radiative transfer and multiple scattering of diffuse ultrasound in polycrystalline media," J. Acoust. Soc. Am. 96, 3675-3683 (1994).

${ }^{12}$ J. A. Turner and R. L. Weaver, "Time dependence of multiply scattered diffuse ultrasound in polycrystalline media," J. Acoust. Soc. Am. 973, 2639-2644 (1995).

${ }^{13}$ J. A. Turner and R. L. Weaver, "Ultrasonic radiative transfer theory: Effects of a fluid-solid interface," J. Acoust. Soc. Am. 98, 2801-2808 (1995)

${ }^{14} \mathrm{M}$. Spies, "Elastic waves in homogeneous and layered transversely isotropic media: Plane waves and Gaussian wave packets. A general approach,' J. Acoust. Soc. Am. 95, 1748-1760 (1994).

${ }^{15}$ F. I. Fedorov, Theory of Elastic Waves in Crystals (Plenum, New York, 1968).

${ }^{16} \mathrm{~J}$. A. Turner, "Elastic wave propagation and scattering in heterogeneous, anisotropic media: Textured polycrystalline materials," Dept. of Engineering Mechanics Technical Report EM1.998 (September 1998).

${ }^{17}$ Matlab, The Language of Technical Computing (The Math Works, Natick, MA, 1996).

${ }^{18} \mathrm{M}$. Spies, "Elastic wave propagation in general transversely isotropic media. I. Green's functions and elastodynamic holography," J. Acoust. Soc. Am. 96, 1144-1157 (1994).

${ }^{19}$ M. Spies, "Elastic wave propagation in transversely isotropic media. II. The generalized Rayleigh function and an integral representation for the transducer field. Theory," J. Acoust. Soc. Am. 97, 1-13 (1995). 